Acta Crystallographica Section C

Crystal Structure

Communications

ISSN 0108-2701

\section{Simple chains in methyl 3,5-dinitro- benzoate, isolated molecules in isopropyl 3,5-dinitrobenzoate, and a three-dimensional framework containing double and sextuple helices in benzyl 3,5-dinitrobenzoate, all at $120 \mathrm{~K}$}

\author{
Thatyana R. A. Vasconcelos, ${ }^{a}$ Marcus V. N. de Souza, ${ }^{a}$ \\ Solange M. S. V. Wardell, ${ }^{\text {a James L. Wardell, }}$, John N. \\ Low $^{c}$ and Christopher Glidewell ${ }^{\mathrm{d} *}$ \\ ${ }^{a}$ Complexo Tecnológico de Medicamentos Farmanguinhos, Av. Comandante \\ Guaranys 447, Jacarepaguá, Rio de Janeiro, RJ, Brazil, 'b Instituto de Química, \\ Departamento de Química Inorgânica, Universidade Federal do Rio de Janeiro, CP \\ 68563, 21945-970 Rio de Janeiro, RJ, Brazil, ' Department of Chemistry, University \\ of Aberdeen, Meston Walk, Old Aberdeen AB24 3UE, Scotland, and ${ }^{\mathbf{d}}$ School of \\ Chemistry, University of St Andrews, Fife KY16 9ST, Scotland \\ Correspondence e-mail: cg@st-andrews.ac.uk
}

Received 21 November 2005

Accepted 22 November 2005

Online 16 December 2005

Molecules of methyl 3,5-dinitrobenzoate, $\mathrm{C}_{8} \mathrm{H}_{6} \mathrm{~N}_{2} \mathrm{O}_{6}$, are linked into $C(7)$ chains by a single nearly linear $\mathrm{C}-\mathrm{H} \cdots \mathrm{O}$ hydrogen bond, but there are no direction-specific interactions in the structure of isopropyl 3,5-dinitrobenzoate, $\mathrm{C}_{10} \mathrm{H}_{10} \mathrm{~N}_{2} \mathrm{O}_{6}$. In benzyl 3,5-dinitrobenzoate, $\mathrm{C}_{14} \mathrm{H}_{10} \mathrm{~N}_{2} \mathrm{O}_{6}$, the molecules are linked by four independent $\mathrm{C}-\mathrm{H} \cdots \mathrm{O}$ hydrogen bonds into a complex three-dimensional framework structure, in which it is possible to identify simple substructures in the form of cyclic centrosymmetric dimers, double helices and sextuple helices.

\section{Comment}

We report here the structures of methyl, isopropyl and benzyl 3,5-dinitrobenzoate, (I)-(III), respectively, derived from lowtemperature diffraction data. It has been recognized since the early days of experimental organic chemistry that the 3,5nitrobenzoate esters of simple alcohols are crystalline solids<smiles>[R]OC(=O)c1cc([N+](=O)[O-])cc([N+](=O)[O-])c1</smiles>

that are very readily purified and crystallized, and indeed these crystalline esters were for many decades utilized as an aid to identification of such alcohols by means of their sharp and characteristic melting temperatures. The structures of a few of these esters have been the subject of isolated reports, including the ethyl ester (Hughes \& Trotter, 1971) and the 2,2dimethylbutyl ester, where $Z^{\prime}=2$ (Sax et al., 1976); both of these structure determinations were based on the use of diffraction data collected at ambient temperature. More recently, the structures of the methyl ester, (I) (Jin \& Xiao, $2000 b$ ), the $n$-propyl ester, where $Z^{\prime}=2$ (Jin \& Xiao, 2005c), and the isopropyl ester, (II) (Jin \& Xiao, 2005a), have been reported, also using diffraction data collected at ambient temperature. The structure of (I) was reported to contain $\pi$-stacked molecules related by translation along [100], with intermolecular C . C c contacts as short as 3.440 (4) A, despite the $a$ repeat vector of 4.5833 (15) $\AA$; no hydrogen bonds were reported in this compound. Although the unit-cell dimensions, the space group and the atomic coordinates for (I) indicate that no phase change has occurred between ambient

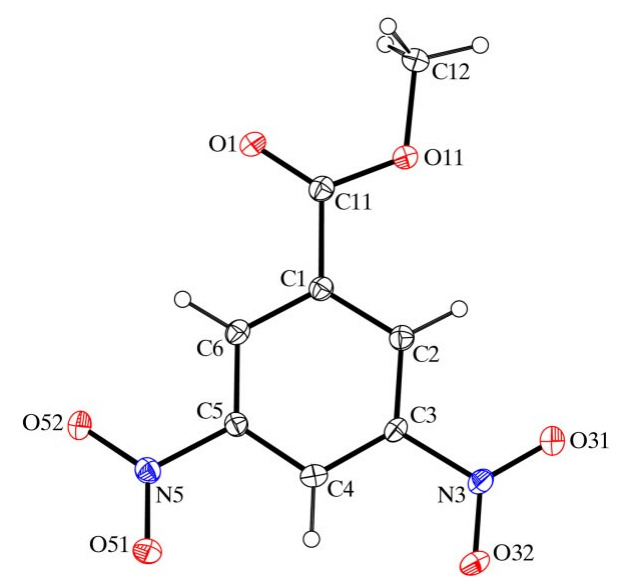

Figure 1

The molecule of (I), showing the atom-labelling scheme. Displacement ellipsoids are drawn at the $30 \%$ probability level.

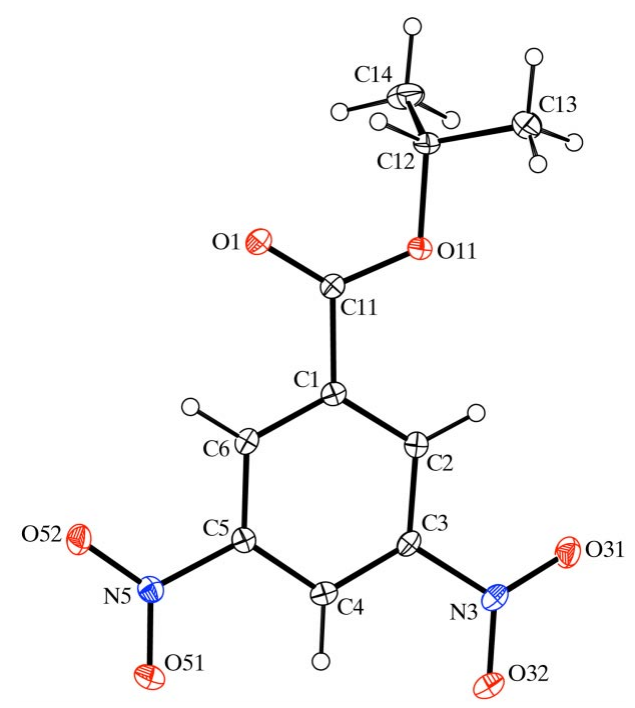

Figure 2

The molecule of (II), showing the atom-labelling scheme. Displacement ellipsoids are drawn at the $30 \%$ probability level. 
temperature and $120 \mathrm{~K}$, we find no $\pi-\pi$ stacking in (I); however, a nearly linear $\mathrm{C}-\mathrm{H} \cdots \mathrm{O}$ hydrogen bond is present, in contrast to the findings reported previously (Jin \& Xiao, 2005b).

The molecule of (I) (Fig. 1) is effectively planar, apart from the $\mathrm{H}$ atoms of the methyl group, as shown by the leading torsion angles (Table 3). In (II) and (III) (Figs. 2 and 3), the ester fragment up to and including atom $\mathrm{C} 12$ is effectively coplanar with the adjacent aryl ring, but in (III) in particular, the remaining torsion angles indicate a markedly non-planar conformation. The bond distances show no unusual values, but in each compound the internal angles at atoms C3 and C5, which are ipso to the nitro groups, are significantly larger than the corresponding angle at $\mathrm{C} 2$, which is ipso to the ester group.

There are no direction-specific intermolecular interactions in the structure of (II), but the molecules of (I) are linked into simple $C(7)$ chains by a single $\mathrm{C}-\mathrm{H} \cdots \mathrm{O}$ hydrogen bond (Table 1). Atom $\mathrm{C} 4$ in the molecule at $(x, y, z)$ acts as a hydrogen-bond donor to carbonyl atom $\mathrm{O} 1$ in the molecule at $\left(\frac{3}{2}+x, \frac{3}{2}-y, \frac{1}{2}+z\right)$, so forming a $C(7)$ (Bernstein et al., 1995) chain running parallel to the [301] direction and generated by the $n$-glide plane at $y=\frac{3}{4}$ (Fig. 4 ). Two such chains, related to one another by inversion, pass through each unit cell, but there are no direction-specific interactions between adjacent chains; in particular, $\mathrm{C}-\mathrm{H} \cdots \pi$ (arene) hydrogen bonds and aromatic $\pi-\pi$ stacking interactions are both absent. The shortest ring-centroid separation, which involves the molecules at $(x, y, z)$ and $\left(\frac{1}{2}+x, \frac{3}{2}-y, \frac{1}{2}+z\right)$, is 5.670 (2) $\AA$ and is clearly too large for effective $\pi-\pi$ stacking.

The molecules of (III) are linked by four independent $\mathrm{C}-$ $\mathrm{H}$... O hydrogen bonds (Table 2) into a three-dimensional framework of considerable complexity. However, it is possible to identify several simple substructures, each generated by a limited number of hydrogen bonds. The formation of the framework is most simply analysed in terms of one finite zero-

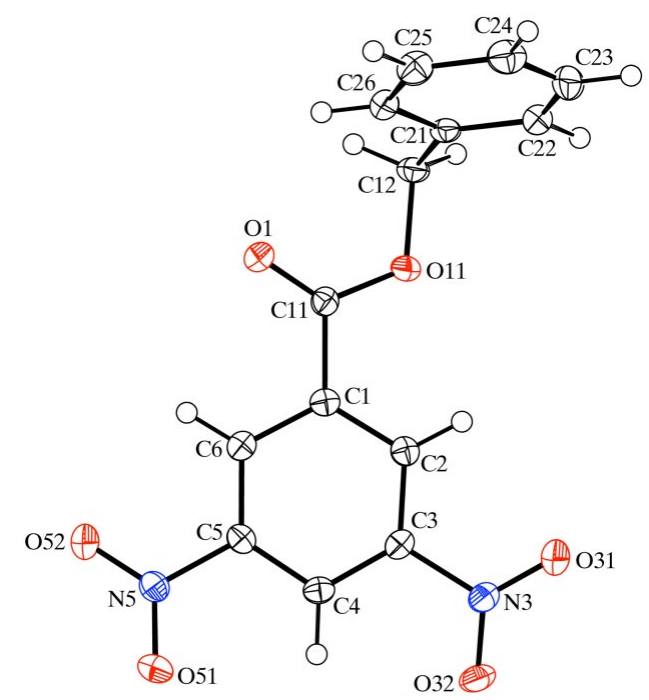

Figure 3

The molecule of (III), showing the atom-labelling scheme. Displacement ellipsoids are drawn at the $30 \%$ probability level. dimensional motif, which can be regarded as the basic building block; this motif is formed by the concerted action of two of the hydrogen bonds and two independent chain motifs, each containing a single hydrogen bond, one of which generates a double helix while the other generates a sextuple helix.

In the first substructure, atoms $\mathrm{C} 6$ and $\mathrm{C} 12$ in the molecule at $(x, y, z)$ act as hydrogen-bond donors, respectively, to atoms $\mathrm{O} 1$ and $\mathrm{O} 52$ in the molecule at $(1-x, 1-y,-z)$, so gener-

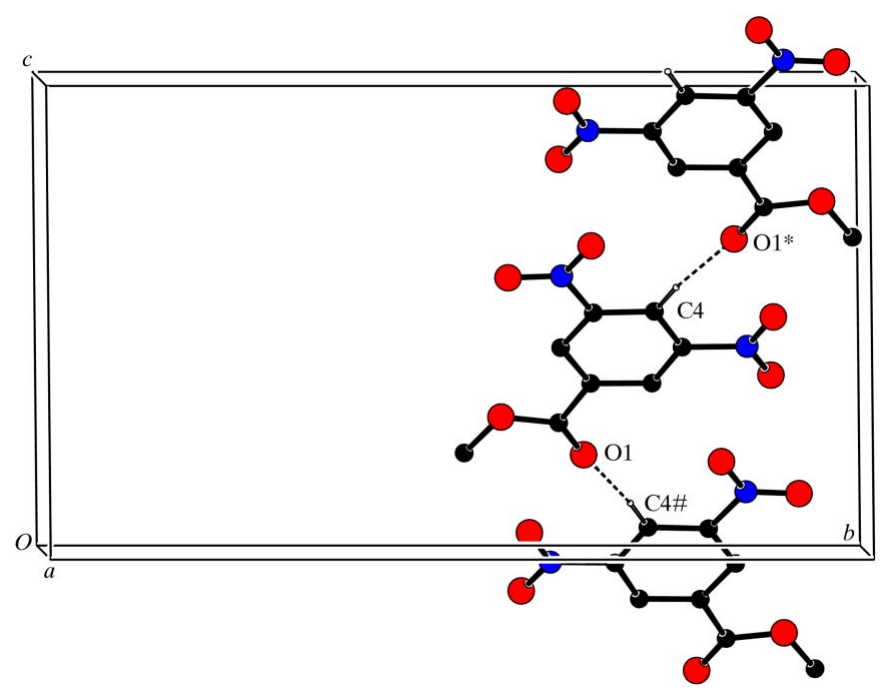

Figure 4

Part of the crystal structure of (I), showing the formation of a $C(7)$ chain along [301]. For the sake of clarity, $\mathrm{H}$ atoms not involved in the motif shown have been omitted. Atoms marked with an asterisk (*) or a hash (\#) are at the symmetry positions $\left(\frac{3}{2}+x, \frac{3}{2}-y, \frac{1}{2}+z\right)$ and $\left(-\frac{3}{2}+x, \frac{3}{2}-y\right.$, $\left.-\frac{1}{2}+z\right)$, respectively.

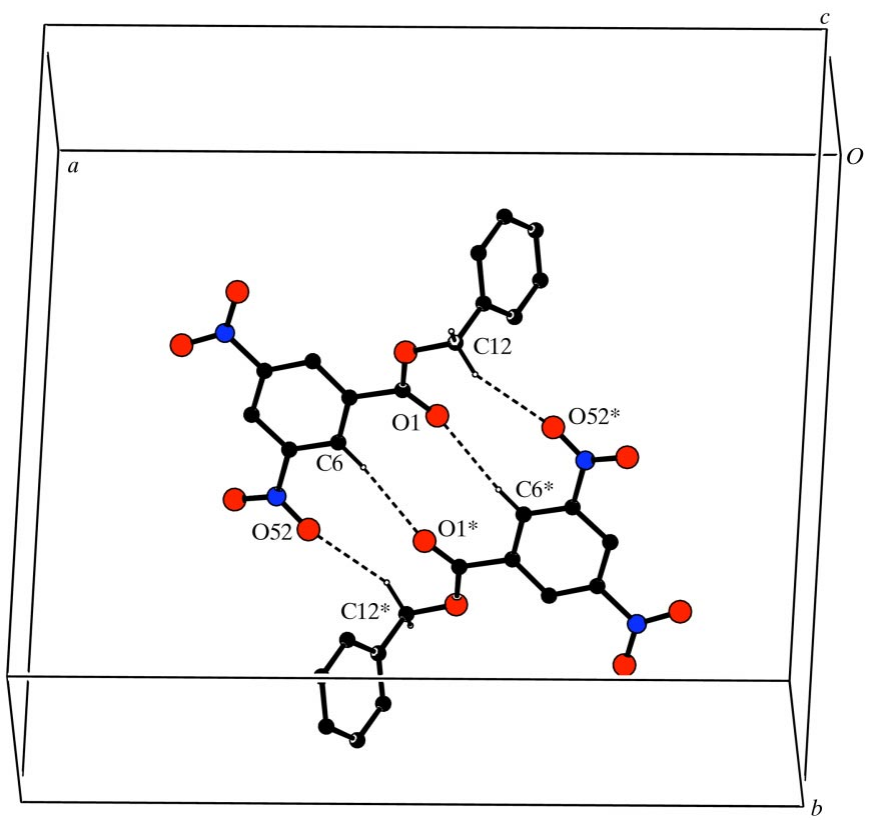

Figure 5

Part of the crystal structure of (III), showing the formation of a centrosymmetric dimer. For the sake of clarity, $\mathrm{H}$ atoms bonded to $\mathrm{C}$ atoms not involved in the motif shown have been omitted. Atoms marked with an asterisk $(*)$ are at the symmetry position $(1-x, 1-y,-z)$. 
ating a centrosymmetric dimer centred at $\left(\frac{1}{2}, \frac{1}{2}, 0\right)$, in which inversion-related pairs of hydrogen bonds generate $R_{2}^{2}(10)$ and $R_{2}^{2}(18)$ rings (Fig. 5). In the second substructure, this time one-dimensional as opposed to zero-dimensional, atom $\mathrm{C} 2$ in the molecule at $(x, y, z)$ acts as a hydrogen-bond donor to atom $\mathrm{O} 32$ in the molecule at $\left(1-y,-\frac{1}{2}+x, \frac{1}{2}+z\right)$, while atom $\mathrm{C} 2$ at $\left(1-y,-\frac{1}{2}+x, \frac{1}{2}+z\right)$ in turn acts as a donor to atom O32 at $\left(\frac{3}{2}-x, \frac{1}{2}-y, 1+z\right)$. Propagation of this hydrogen bond thus generates a $C(5)$ helical chain running parallel to the [001] direction and generated by the $4_{2}$ screw axis along $\left(\frac{3}{4}, \frac{1}{4}, z\right)$ (Fig. 6). Because the screw axis is of the $4_{2}$ type, this chain links the molecules at $(x, y, z)$ and $(x, y, 2+z)$, so that complete definition of this substructure requires two coaxial helices offset by a unit translation along [001].

The combination of this helical chain (Fig. 6) with the centrosymmetric dimer motif (Fig. 5) then links each helical chain to four adjacent helical chains; for example, the helix along $\left(\frac{3}{4}, \frac{1}{4}, z\right)$ is directly linked in this way to those along
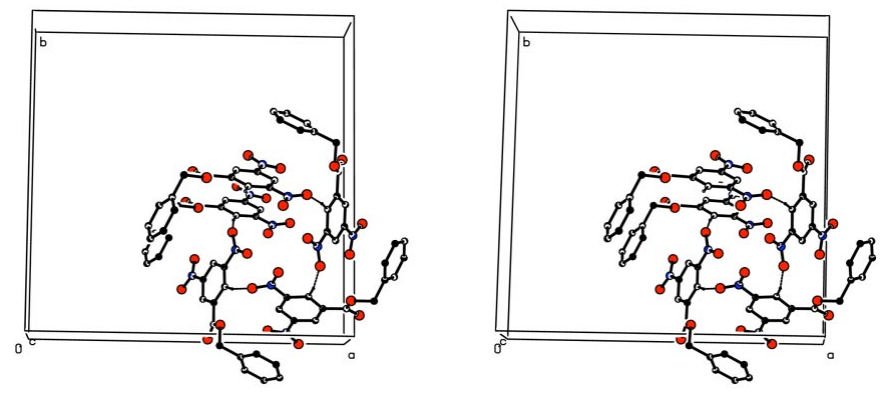

Figure 6

A stereoview of part of the crystal structure of (III), showing a hydrogenbonded helical $C(5)$ chain generated by the $4_{2}$ screw axis along $\left(\frac{3}{4}, \frac{1}{4}, z\right)$ and forming one strand of a double helix. For the sake of clarity, $\mathrm{H}$ atoms not involved in the motif shown have been omitted.
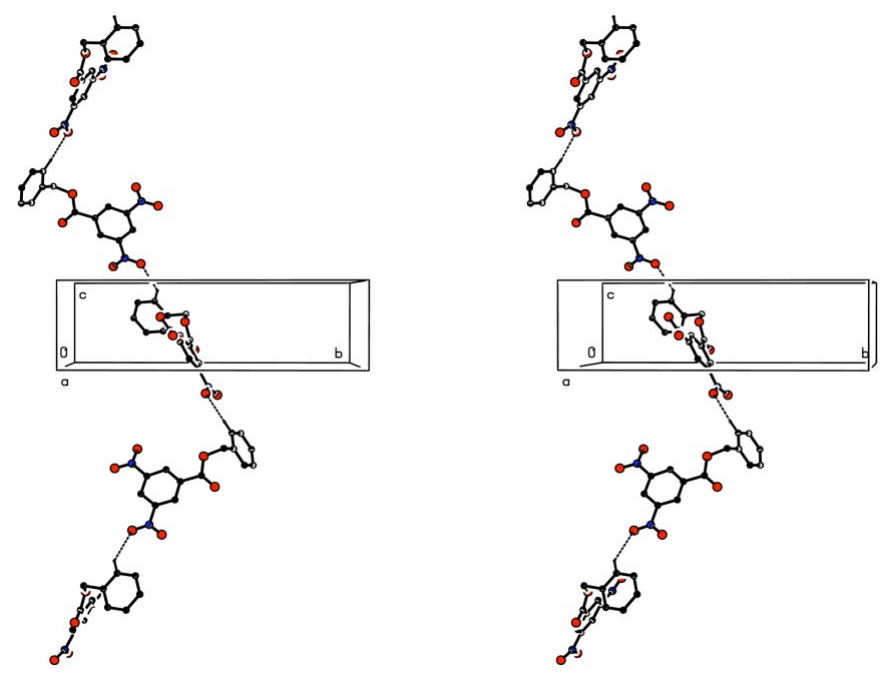

Figure 7

A stereoview of part of the crystal structure of (III), showing a hydrogenbonded helical $C(11)$ chain generated by the $4_{2}$ screw axis along $\left(\frac{3}{4}, \frac{1}{4}, z\right)$ and forming one strand of a sextuple helix. For the sake of clarity, $\mathrm{H}$ atoms not involved in the motif shown have been omitted.

$\left(\frac{1}{4}, \frac{3}{4}, z\right),\left(\frac{5}{4}, \frac{3}{4}, z\right),\left(\frac{1}{4},-\frac{1}{4}, z\right)$ and $\left(\frac{5}{4},-\frac{1}{4}, z\right)$, so forming a continuous three-dimensional framework. Because of the double-helical nature of the [001] chain, there are in fact two such frameworks, intimately interwoven.

In addition, there is a fourth $\mathrm{C}-\mathrm{H} \cdots \mathrm{O}$ hydrogen bond, whose action in isolation is to generate a sextuple helix of $C$ (11) chains (Fig. 7), but which in combination with the first chain-forming hydrogen bond links the two interwoven frameworks into a single continuous structure. Atom C22 in the molecule at $(x, y, z)$ acts as a hydrogen-bond donor to atom $\mathrm{O} 51$ in the molecule at $\left(1-y,-\frac{1}{2}+x, \frac{3}{2}+z\right)$, and atom $\mathrm{C} 22$ at $\left(1-y,-\frac{1}{2}+x, \frac{3}{2}+z\right)$ likewise acts as a donor to atom O51 at $\left(\frac{3}{2}-x, \frac{1}{2}-y, 3+z\right)$, and thence via $\left(\frac{1}{2}+y, 1-x, \frac{9}{2}+z\right)$ to $(x, y, 6+z)$, so generating the sextuple helix. At the same time, atom $\mathrm{C} 2$ in the molecule at $\left(1-y,-\frac{1}{2}+x, \frac{3}{2}+z\right)$ acts as a hydrogen-bond donor to atom $\mathrm{O} 32$ in the molecule at $\left(\frac{3}{2}-x\right.$, $\left.\frac{1}{2}-y, 2+z\right)$, thereby linking the two coaxial $C(5)$ helices along $\left(\frac{3}{4}, \frac{1}{4}, z\right)$ and hence linking the two frameworks.

\section{Experimental}

Samples of compounds (I)-(III) were prepared from 3,5-dinitrobenzoic acid according to a published procedure (Vogel, 1977). The compounds had the expected NMR and IR spectra, and the melting points were in agreement with those reported previously (Vogel, 1977). Crystals suitable for single-crystal X-ray diffraction were grown by slow evaporation of solutions in ethanol.

\section{Compound (I)}

\section{Crystal data}

$\mathrm{C}_{8} \mathrm{H}_{6} \mathrm{~N}_{2} \mathrm{O}_{6}$

$M_{r}=226.15$

Monoclinic, $P 2_{1} / n$

$a=4.5664$ (4) ̊

$b=18.727$ (2) $\AA$

$c=10.8416(10) \AA$

$\beta=101.787(6)^{\circ}$

$V=907.57(15) \AA^{3}$

$Z=4$

\section{Data collection}

Nonius KappaCCD diffractometer $\varphi$ and $\omega$ scans

Absorption correction: multi-scan (SADABS; Sheldrick, 2003)

$T_{\min }=0.935, T_{\max }=0.997$

9551 measured reflections

2070 independent reflections

\section{Refinement}

Refinement on $F^{2}$

$R\left[F^{2}>2 \sigma\left(F^{2}\right)\right]=0.058$

$w R\left(F^{2}\right)=0.174$

$S=1.09$

2070 reflections

146 parameters

$\mathrm{H}$-atom parameters constrained

Table 1

Hydrogen-bond geometry $\left(\AA,^{\circ}\right)$ for (I).

\begin{tabular}{lllll}
\hline$D-\mathrm{H} \cdots A$ & $D-\mathrm{H}$ & $\mathrm{H} \cdots A$ & $D \cdots A$ & $D-\mathrm{H} \cdots A$ \\
\hline $\mathrm{C} 4-\mathrm{H} 4 \cdots \mathrm{O} 1^{\mathrm{i}}$ & 0.95 & 2.46 & $3.399(3)$ & 171 \\
\hline
\end{tabular}

Symmetry code: (i) $x+\frac{3}{2},-y+\frac{3}{2}, z+\frac{1}{2}$.

\author{
$D_{x}=1.655 \mathrm{Mg} \mathrm{m}^{-3}$ \\ Mo $K \alpha$ radiation \\ Cell parameters from 2070 \\ reflections \\ $\theta=2.9-27.6^{\circ}$ \\ $\mu=0.14 \mathrm{~mm}^{-1}$ \\ $T=120$ (2) K \\ Lath, colourless \\ $0.52 \times 0.12 \times 0.02 \mathrm{~mm}$
}

1319 reflections with $I>2 \sigma(I)$

$R_{\text {int }}=0.060$

$\theta_{\max }=27.6^{\circ}$

$h=-5 \rightarrow 5$

$k=-24 \rightarrow 24$

$l=-14 \rightarrow 14$

$$
\begin{gathered}
w=1 /\left[\sigma^{2}\left(F_{\mathrm{o}}^{2}\right)+(0.0823 P)^{2}\right. \\
\quad+0.2307 P] \\
\text { where } P=\left(F_{\mathrm{o}}^{2}+2 F_{\mathrm{c}}^{2}\right) / 3 \\
(\Delta / \sigma)_{\max }<0.001 \\
\Delta \rho_{\max }=0.25 \AA^{-3} \\
\Delta \rho_{\min }=-0.29 \mathrm{e}^{-3}
\end{gathered}
$$




\section{Compound (II)}

Crystal data
$\mathrm{C}_{10} \mathrm{H}_{10} \mathrm{~N}_{2} \mathrm{O}_{6}$

$M_{r}=254.20$

Monoclinic, $P 2_{1} / n$

$a=9.7037$ (3) $\AA$

$b=5.7152(2) \AA$

$c=20.4739$ (9) $\AA$

$\beta=95.504(2)^{\circ}$

$V=1130.22(7) \AA^{3}$

$Z=4$

$D_{x}=1.494 \mathrm{Mg} \mathrm{m}^{-3}$
Mo $K \alpha$ radiation
Cell parameters from 2596
$\quad$ reflections
$\theta=3.8-27.5^{\circ}$
$\mu=0.13 \mathrm{~mm}^{-1}$
$T=120(2) \mathrm{K}$
Block, colourless
$0.42 \times 0.40 \times 0.38 \mathrm{~mm}$

Data collection

Nonius KappaCCD diffractometer $\varphi$ and $\omega$ scans

Absorption correction: multi-scan

(SADABS; Sheldrick, 2003)

$T_{\min }=0.944, T_{\max }=0.954$

10022 measured reflections

\section{Refinement}

Refinement on $F^{2}$

$R\left[F^{2}>2 \sigma\left(F^{2}\right)\right]=0.039$

$w R\left(F^{2}\right)=0.105$

$S=1.05$

2596 reflections

165 parameters

$\mathrm{H}$-atom parameters constrained

\section{Table 2}

Hydrogen-bond geometry $\left(\AA,^{\circ}\right)$ for (III).

\begin{tabular}{lllll}
\hline$D-\mathrm{H} \cdots A$ & $D-\mathrm{H}$ & $\mathrm{H} \cdots A$ & $D \cdots A$ & $D-\mathrm{H} \cdots A$ \\
\hline $\mathrm{C} 2-\mathrm{H} 2 \cdots \mathrm{O} 32^{\mathrm{ii}}$ & 0.95 & 2.52 & $3.460(2)$ & 170 \\
$\mathrm{C} 6-\mathrm{H} 6 \cdots \mathrm{O} 1^{\mathrm{iii}}$ & 0.95 & 2.57 & $3.493(2)$ & 164 \\
$\mathrm{C} 12-\mathrm{H} 12 A \cdots \mathrm{O} 52^{\mathrm{iii}}$ & 0.99 & 2.56 & $3.474(2)$ & 154 \\
$\mathrm{C} 22-\mathrm{H} 22 \cdots \mathrm{O} 51^{\text {iv }}$ & 0.95 & 2.57 & $3.360(2)$ & 140 \\
\hline
\end{tabular}

Symmetry codes: (ii) $\quad-y+1, x-\frac{1}{2}, z+\frac{1}{2}$; $\quad$ (iii) $\quad-x+1,-y+1,-z$; $\quad$ (iv)

$-y+1, x-\frac{1}{2}, z+\frac{3}{2}$

\section{Compound (III)}

\section{Crystal data}

$\mathrm{C}_{14} \mathrm{H}_{10} \mathrm{~N}_{2} \mathrm{O}_{6}$
$M_{r}=302.24$
Tetragonal, $P 4_{2} / n$
$a=20.8531(5) \AA$
$c=6.0377(2) \AA$
$V=2625.50(12) \AA^{3}$
$Z=8$
$D_{x}=1.529 \mathrm{Mg} \mathrm{m}^{-3}$

Mo $K \alpha$ radiation

Cell parameters from 3000 reflections

$\theta=3.1-27.5^{\circ}$

$\mu=0.12 \mathrm{~mm}^{-1}$

$T=120(2) \mathrm{K}$

Needle, colourless

$0.22 \times 0.06 \times 0.04 \mathrm{~mm}$

\section{Data collection}

Nonius KappaCCD diffractometer $\varphi$ and $\omega$ scans

Absorption correction: multi-scan

(SADABS; Sheldrick, 2003)

$T_{\min }=0.978, T_{\max }=0.995$

18873 measured reflections

\section{Refinement}

Refinement on $F^{2}$

$R\left[F^{2}>2 \sigma\left(F^{2}\right)\right]=0.043$

$w R\left(F^{2}\right)=0.103$

$S=1.05$

199 parameters

3000 independent reflections 2413 reflections with $I>2 \sigma(I)$

$R_{\text {int }}=0.053 ; \theta_{\max }=27.5^{\circ}$

$h=-27 \rightarrow 26$

$k=-16 \rightarrow 27$

$l=-7 \rightarrow 7$

$w=1 /\left[\sigma^{2}\left(F_{\mathrm{o}}^{2}\right)+(0.0355 P)^{2}\right.$ $+1.5651 P]$ where $P=\left(F_{\mathrm{o}}^{2}+2 F_{\mathrm{c}}^{2}\right) / 3$

$(\Delta / \sigma)_{\max }=0.001$

$\Delta \rho_{\max }=0.24{\mathrm{e} \AA^{-3}}^{-3}$

$\Delta \rho_{\min }=-0.23 \mathrm{e}^{-3}$
3000 reflections

$\mathrm{H}$-atom parameters constrained

Table 3

Selected angles and torsion angles $\left({ }^{\circ}\right)$ for compounds (I)-(III).

\begin{tabular}{lccc}
\hline & (I) & (II) & (III) \\
\hline $\mathrm{C} 6-\mathrm{C} 1-\mathrm{C} 2$ & $120.2(2)$ & $120.34(11)$ & $120.82(14)$ \\
$\mathrm{C} 2-\mathrm{C} 3-\mathrm{C} 4$ & $123.3(2)$ & $123.34(11)$ & $122.94(15)$ \\
$\mathrm{C} 4-\mathrm{C} 5-\mathrm{C} 6$ & $123.3(2)$ & $122.58(12)$ & $123.37(15)$ \\
$\mathrm{C} 2-\mathrm{C} 1-\mathrm{C} 11-\mathrm{O} 11$ & $2.0(3)$ & $-6.24(16)$ & $15.2(2)$ \\
$\mathrm{C} 1-\mathrm{C} 11-\mathrm{O} 11-\mathrm{C} 12$ & $179.74(19)$ & $-176.88(9)$ & $177.03(12)$ \\
$\mathrm{C} 11-\mathrm{O} 11-\mathrm{C} 12-\mathrm{C} 13$ & - & $152.12(12)$ & - \\
$\mathrm{C} 11-\mathrm{O} 11-\mathrm{C} 12-\mathrm{C} 14$ & - & $-85.33(13)$ & - \\
$\mathrm{C} 11-\mathrm{O} 11-\mathrm{C} 12-\mathrm{C} 21$ & - & - & $93.65(16)$ \\
$\mathrm{O} 11-\mathrm{C} 12-\mathrm{C} 21-\mathrm{C} 22$ & - & - & $89.93(7)$ \\
$\mathrm{C} 2-\mathrm{C} 3-\mathrm{N} 3-\mathrm{O} 31$ & $0.2(3)$ & $-4.88(16)$ & $9.1(2)$ \\
$\mathrm{C} 4-\mathrm{C} 5-\mathrm{N} 5-\mathrm{O} 51$ & $4.2(3)$ & $6.13(16)$ & $7.5(2)$ \\
\hline
\end{tabular}

For each of compounds (I) and (II), the space group $P 2_{1} / n$ was uniquely assigned from the systematic absences. For compound (III), the space group $P 4_{2} / n$ was uniquely assigned from the systematic absences, and the setting adopted had the origin coincident with a centre of inversion. All $\mathrm{H}$ atoms were located in difference maps and then treated as riding atoms, with $\mathrm{C}-\mathrm{H}$ distances of 0.95 (aromatic), 0.98 (methyl), $0.99\left(\mathrm{CH}_{2}\right)$ or $1.00 \AA$ (aliphatic $\mathrm{CH}$ ), and with $U_{\text {iso }}(\mathrm{H})$ values of $1.2 U_{\mathrm{eq}}(\mathrm{C})$, or $1.5 U_{\mathrm{eq}}(\mathrm{C})$ for the methyl groups.

For all compounds, data collection: COLLECT (Hooft, 1999); cell refinement: DENZO (Otwinowski \& Minor, 1997) and COLLECT; data reduction: DENZO and COLLECT; program(s) used to solve structure: OSCAIL (McArdle, 2003) and SHELXS97 (Sheldrick, 1997); program(s) used to refine structure: OSCAIL and SHELXL97 (Sheldrick, 1997); molecular graphics: PLATON (Spek, 2003); software used to prepare material for publication: SHELXL97 and PRPKAPPA (Ferguson, 1999).

X-ray data were collected at the EPSRC X-ray Crystallographic Service, University of Southampton, England; the authors thank the staff for all their help and advice. JLW and SMSVW thank CNPq and FAPERJ for financial support.

Supplementary data for this paper are available from the IUCr electronic archives (Reference: SK1889). Services for accessing these data are described at the back of the journal.

\section{References}

Bernstein, J., Davis, R. E., Shimoni, L. \& Chang, N.-L. (1995). Angew. Chem. Int. Ed. Engl. 34, 1555-1573.

Ferguson, G. (1999). PRPKAPPA. University of Guelph, Canada.

Hooft, R. W. W. (1999). COLLECT. Nonius BV, Delft, The Netherlands.

Hughes, D. L. \& Trotter, J. (1971). J. Chem. Soc. A, pp. 2358-2361.

Jin, L.-F. \& Xiao, F.-P. (2005a). Acta Cryst. E61, o1269-o1270.

Jin, L.-F. \& Xiao, F.-P. (2005b). Acta Cryst. E61, o1276-o1277.

Jin, L.-F. \& Xiao, F.-P. (2005c). Acta Cryst. E61, o1826-o1827.

McArdle, P. (2003). OSCAIL for Windows. Version 10. Crystallography Centre, Chemistry Department, NUI Galway, Ireland.

Otwinowski, Z. \& Minor, W. (1997). Methods in Enzymology, Vol. 276, Macromolecular Crystallography, Part A, edited by C. W. Carter Jr \& R. M. Sweet, pp. 307-326. New York: Academic Press.

Sax, M., Rodrigues, M., Blank, G., Wood, M. K. \& Pletcher, J. (1976). Acta Cryst. B32, 1953-1956.

Sheldrick, G. M. (1997). SHELXS97 and SHELXL97. University of Göttingen, Germany.

Sheldrick, G. M. (2003). SADABS. Version 2.10. University of Göttingen, Germany.

Spek, A. L. (2003). J. Appl. Cryst. 36, 7-13.

Vogel, A. I. (1977). Elementary Practical Organic Chemistry, Part 2, Qualitative Organic Analysis, 2nd ed., p. 75. London: Longman. 


\section{supporting information}

Acta Cryst. (2006). C62, o26-o29 [doi:10.1107/S0108270105038631]

Simple chains in methyl 3,5-dinitrobenzoate, isolated molecules in isopropyl 3,5-dinitrobenzoate, and a three-dimensional framework containing double and sextuple helices in benzyl 3,5-dinitrobenzoate, all at $120 \mathrm{~K}$

Thatyana R. A. Vasconcelos, Marcus V. N. de Souza, Solange M. S. V. Wardell, James L. Wardell, John N. Low and Christopher Glidewell

Computing details

For all compounds, data collection: COLLECT (Hooft, 1999); cell refinement: DENZO (Otwinowski \& Minor, 1997) and COLLECT; data reduction: DENZO and COLLECT; program(s) used to solve structure: OSCAIL (McArdle, 2003) and SHELXS97 (Sheldrick, 1997); program(s) used to refine structure: OSCAIL and SHELXL97 (Sheldrick, 1997); molecular graphics: PLATON (Spek, 2003); software used to prepare material for publication: SHELXL97 and PRPKAPPA

(Ferguson, 1999).

(I) Methyl 3,5-dinitrobenzoate

Crystal data

$\mathrm{C}_{8} \mathrm{H}_{6} \mathrm{~N}_{2} \mathrm{O}_{6}$

$M_{r}=226.15$

$F(000)=464$

$D_{\mathrm{x}}=1.655 \mathrm{Mg} \mathrm{m}^{-3}$

Monoclinic, $P 2_{1} / n$

Mo $K \alpha$ radiation, $\lambda=0.71073 \AA$

Hall symbol: -P 2 yn

Cell parameters from 2070 reflections

$a=4.5664$ (4) $\AA$

$\theta=2.9-27.6^{\circ}$

$b=18.727(2) \AA$

$c=10.8416(10) \AA$

$\beta=101.787(6)^{\circ}$

$V=907.57(15) \AA^{3}$

$\mu=0.15 \mathrm{~mm}^{-1}$

$T=120 \mathrm{~K}$

Lath, colourless

$Z=4$

$0.52 \times 0.12 \times 0.02 \mathrm{~mm}$

Data collection

Nonius KappaCCD

diffractometer

Radiation source: Bruker-Nonius FR91 rotating anode

Graphite monochromator

Detector resolution: 9.091 pixels $\mathrm{mm}^{-1}$

$\varphi$ and $\omega$ scans

Absorption correction: multi-scan

(SADABS; Sheldrick, 2003)

$T_{\min }=0.935, T_{\max }=0.997$

9551 measured reflections

2070 independent reflections

1319 reflections with $I>2 \sigma(I)$

$R_{\text {int }}=0.060$

$\theta_{\max }=27.6^{\circ}, \theta_{\min }=2.9^{\circ}$

$h=-5 \rightarrow 5$

$k=-24 \rightarrow 24$

$l=-14 \rightarrow 14$ 


\section{Refinement}

Refinement on $F^{2}$

Least-squares matrix: full

$R\left[F^{2}>2 \sigma\left(F^{2}\right)\right]=0.058$

$w R\left(F^{2}\right)=0.174$

$S=1.09$

2070 reflections

146 parameters

0 restraints

Primary atom site location: structure-invariant direct methods
Secondary atom site location: difference Fourier map

Hydrogen site location: inferred from neighbouring sites

$\mathrm{H}$-atom parameters constrained

$w=1 /\left[\sigma^{2}\left(F_{\mathrm{o}}{ }^{2}\right)+(0.0823 P)^{2}+0.2307 P\right]$ where $P=\left(F_{\mathrm{o}}^{2}+2 F_{\mathrm{c}}^{2}\right) / 3$

$(\Delta / \sigma)_{\max }<0.001$

$\Delta \rho_{\max }=0.25{\mathrm{e} \AA^{-3}}^{-3}$

$\Delta \rho_{\min }=-0.29$ e $\AA^{-3}$

Fractional atomic coordinates and isotropic or equivalent isotropic displacement parameters $\left(\AA^{2}\right)$

\begin{tabular}{lllll}
\hline & $x$ & $y$ & $z$ & $U_{\text {iso }} / U_{\text {eq }}$ \\
\hline O1 & $-0.2774(3)$ & $0.67000(9)$ & $0.18351(15)$ & $0.0278(4)$ \\
O11 & $-0.0789(4)$ & $0.56667(9)$ & $0.26896(16)$ & $0.0297(5)$ \\
O31 & $0.8123(5)$ & $0.56191(10)$ & $0.58930(19)$ & $0.0462(6)$ \\
O32 & $1.0328(4)$ & $0.65946(10)$ & $0.66327(15)$ & $0.0315(5)$ \\
O51 & $0.6277(4)$ & $0.88672(9)$ & $0.50125(17)$ & $0.0356(5)$ \\
O52 & $0.2069(4)$ & $0.89026(9)$ & $0.36595(17)$ & $0.0343(5)$ \\
N3 & $0.8306(5)$ & $0.62668(11)$ & $0.59376(18)$ & $0.0270(5)$ \\
N5 & $0.4141(4)$ & $0.85809(11)$ & $0.43276(18)$ & $0.0251(5)$ \\
C1 & $0.1600(5)$ & $0.67207(13)$ & $0.3470(2)$ & $0.0214(5)$ \\
C2 & $0.3754(5)$ & $0.63231(13)$ & $0.4289(2)$ & $0.0230(6)$ \\
C3 & $0.6005(5)$ & $0.66864(13)$ & $0.5090(2)$ & $0.0218(5)$ \\
C4 & $0.6224(5)$ & $0.74254(13)$ & $0.5126(2)$ & $0.0224(5)$ \\
C5 & $0.4021(5)$ & $0.77944(13)$ & $0.4308(2)$ & $0.0215(5)$ \\
C6 & $0.1734(5)$ & $0.74635(13)$ & $0.3480(2)$ & $0.0228(5)$ \\
C11 & $-0.0892(5)$ & $0.63688(13)$ & $0.2566(2)$ & $0.0233(6)$ \\
C12 & $-0.3115(6)$ & $0.52557(14)$ & $0.1862(2)$ & $0.0333(7)$ \\
H2 & 0.3673 & 0.5816 & 0.4295 & $0.028^{*}$ \\
H4 & 0.7799 & 0.7664 & 0.5681 & $0.027^{*}$ \\
H6 & 0.0277 & 0.7737 & 0.2926 & $0.027^{*}$ \\
H12A & -0.5082 & 0.5398 & 0.2008 & $0.050^{*}$ \\
H12B & -0.2803 & 0.4745 & 0.2042 & $0.050^{*}$ \\
H12C & -0.3020 & 0.5348 & 0.0982 & $0.050^{*}$ \\
& & & & \\
\hline
\end{tabular}

Atomic displacement parameters $\left(\AA^{2}\right)$

\begin{tabular}{lllllll}
\hline & $U^{11}$ & $U^{22}$ & $U^{33}$ & $U^{12}$ & $U^{13}$ & $U^{23}$ \\
\hline O1 & $0.0261(9)$ & $0.0272(10)$ & $0.0266(9)$ & $0.0019(7)$ & $-0.0030(8)$ & $0.0002(7)$ \\
O11 & $0.0302(10)$ & $0.0234(10)$ & $0.0302(10)$ & $-0.0011(7)$ & $-0.0062(8)$ & $0.0004(7)$ \\
O31 & $0.0555(13)$ & $0.0244(11)$ & $0.0479(12)$ & $0.0059(9)$ & $-0.0151(10)$ & $0.0002(9)$ \\
O32 & $0.0265(10)$ & $0.0376(11)$ & $0.0259(9)$ & $0.0003(8)$ & $-0.0052(8)$ & $0.0009(8)$ \\
O51 & $0.0334(11)$ & $0.0275(10)$ & $0.0401(11)$ & $-0.0079(8)$ & $-0.0058(9)$ & $0.0009(9)$ \\
O52 & $0.0358(11)$ & $0.0244(10)$ & $0.0378(10)$ & $0.0085(8)$ & $-0.0040(9)$ & $0.0004(8)$ \\
N3 & $0.0277(12)$ & $0.0283(13)$ & $0.0224(10)$ & $0.0037(9)$ & $-0.0006(9)$ & $0.0010(9)$ \\
N5 & $0.0276(12)$ & $0.0230(11)$ & $0.0253(10)$ & $-0.0014(9)$ & $0.0065(9)$ & $0.0000(9)$
\end{tabular}


supporting information

\begin{tabular}{lllllll} 
C1 & $0.0229(12)$ & $0.0231(13)$ & $0.0184(11)$ & $0.0003(9)$ & $0.0048(10)$ & $0.0009(9)$ \\
C2 & $0.0254(13)$ & $0.0236(13)$ & $0.0203(11)$ & $0.0013(10)$ & $0.0056(10)$ & $0.0003(10)$ \\
C3 & $0.0196(12)$ & $0.0248(13)$ & $0.0198(11)$ & $0.0039(10)$ & $0.0012(10)$ & $0.0028(10)$ \\
C4 & $0.0197(12)$ & $0.0278(14)$ & $0.0198(12)$ & $-0.0015(10)$ & $0.0039(9)$ & $-0.0022(10)$ \\
C6 & $0.0229(12)$ & $0.0258(13)$ & $0.0196(11)$ & $0.0029(10)$ & $0.0041(10)$ & $0.0016(10)$ \\
C5 & $0.0236(13)$ & $0.0200(13)$ & $0.0213(12)$ & $0.0005(10)$ & $0.0053(10)$ & $0.0000(10)$ \\
C11 & $0.0247(13)$ & $0.0260(13)$ & $0.0197(11)$ & $0.0024(10)$ & $0.0057(10)$ & $0.0000(10)$ \\
C12 & $0.0353(16)$ & $0.0242(14)$ & $0.0346(15)$ & $-0.0042(11)$ & $-0.0065(12)$ & $-0.0022(11)$ \\
\hline
\end{tabular}

Geometric parameters $\left(\AA,{ }^{\circ}\right)$

\begin{tabular}{|c|c|c|c|}
\hline $\mathrm{C} 1-\mathrm{C} 6$ & $1.392(3)$ & $\mathrm{C} 3-\mathrm{C} 4$ & $1.387(3)$ \\
\hline $\mathrm{C} 1-\mathrm{C} 2$ & $1.398(3)$ & $\mathrm{C} 3-\mathrm{N} 3$ & $1.474(3)$ \\
\hline $\mathrm{C} 1-\mathrm{C} 11$ & $1.495(3)$ & $\mathrm{N} 3-\mathrm{O} 31$ & $1.216(3)$ \\
\hline $\mathrm{C} 11-\mathrm{O} 1$ & $1.214(3)$ & $\mathrm{N} 3-\mathrm{O} 32$ & $1.230(2)$ \\
\hline $\mathrm{C} 11-\mathrm{O} 11$ & $1.322(3)$ & $\mathrm{C} 4-\mathrm{C} 5$ & $1.383(3)$ \\
\hline $\mathrm{O} 11-\mathrm{C} 12$ & $1.462(3)$ & $\mathrm{C} 4-\mathrm{H} 4$ & 0.95 \\
\hline $\mathrm{C} 12-\mathrm{H} 12 \mathrm{~A}$ & 0.98 & $\mathrm{C} 6-\mathrm{C} 5$ & $1.378(3)$ \\
\hline $\mathrm{C} 12-\mathrm{H} 12 \mathrm{~B}$ & 0.98 & $\mathrm{C} 6-\mathrm{H} 6$ & 0.95 \\
\hline $\mathrm{C} 12-\mathrm{H} 12 \mathrm{C}$ & 0.98 & $\mathrm{C} 5-\mathrm{N} 5$ & $1.474(3)$ \\
\hline $\mathrm{C} 2-\mathrm{C} 3$ & $1.381(3)$ & $\mathrm{N} 5-\mathrm{O} 51$ & $1.223(2)$ \\
\hline $\mathrm{C} 2-\mathrm{H} 2$ & 0.95 & $\mathrm{~N} 5-\mathrm{O} 52$ & $1.226(2)$ \\
\hline $\mathrm{C} 6-\mathrm{C} 1-\mathrm{C} 2$ & $120.2(2)$ & $\mathrm{C} 2-\mathrm{C} 3-\mathrm{N} 3$ & $118.3(2)$ \\
\hline $\mathrm{C} 6-\mathrm{C} 1-\mathrm{C} 11$ & $118.2(2)$ & $\mathrm{C} 4-\mathrm{C} 3-\mathrm{N} 3$ & $118.4(2)$ \\
\hline $\mathrm{C} 2-\mathrm{C} 1-\mathrm{C} 11$ & $121.6(2)$ & $\mathrm{O} 31-\mathrm{N} 3-\mathrm{O} 32$ & $124.0(2)$ \\
\hline $\mathrm{O} 1-\mathrm{C} 11-\mathrm{O} 11$ & $125.5(2)$ & $\mathrm{O} 31-\mathrm{N} 3-\mathrm{C} 3$ & $118.2(2)$ \\
\hline $\mathrm{O} 1-\mathrm{C} 11-\mathrm{C} 1$ & $123.1(2)$ & $\mathrm{O} 32-\mathrm{N} 3-\mathrm{C} 3$ & $117.8(2)$ \\
\hline $\mathrm{O} 11-\mathrm{C} 11-\mathrm{C} 1$ & $111.44(19)$ & $\mathrm{C} 5-\mathrm{C} 4-\mathrm{C} 3$ & $116.2(2)$ \\
\hline $\mathrm{C} 11-\mathrm{O} 11-\mathrm{C} 12$ & $117.08(18)$ & $\mathrm{C} 5-\mathrm{C} 4-\mathrm{H} 4$ & 121.9 \\
\hline $\mathrm{O} 11-\mathrm{C} 12-\mathrm{H} 12 \mathrm{~A}$ & 109.5 & $\mathrm{C} 3-\mathrm{C} 4-\mathrm{H} 4$ & 121.9 \\
\hline $\mathrm{O} 11-\mathrm{C} 12-\mathrm{H} 12 \mathrm{~B}$ & 109.5 & $\mathrm{C} 5-\mathrm{C} 6-\mathrm{C} 1$ & $118.7(2)$ \\
\hline $\mathrm{H} 12 \mathrm{~A}-\mathrm{C} 12-\mathrm{H} 12 \mathrm{~B}$ & 109.5 & $\mathrm{C} 5-\mathrm{C} 6-\mathrm{H} 6$ & 120.6 \\
\hline $\mathrm{O} 11-\mathrm{C} 12-\mathrm{H} 12 \mathrm{C}$ & 109.5 & $\mathrm{C} 1-\mathrm{C} 6-\mathrm{H} 6$ & 120.6 \\
\hline $\mathrm{H} 12 \mathrm{~A}-\mathrm{C} 12-\mathrm{H} 12 \mathrm{C}$ & 109.5 & $\mathrm{C} 4-\mathrm{C} 5-\mathrm{C} 6$ & $123.3(2)$ \\
\hline $\mathrm{H} 12 \mathrm{~B}-\mathrm{C} 12-\mathrm{H} 12 \mathrm{C}$ & 109.5 & $\mathrm{C} 6-\mathrm{C} 5-\mathrm{N} 5$ & $118.7(2)$ \\
\hline $\mathrm{C} 3-\mathrm{C} 2-\mathrm{C} 1$ & $118.3(2)$ & $\mathrm{C} 4-\mathrm{C} 5-\mathrm{N} 5$ & $118.1(2)$ \\
\hline $\mathrm{C} 3-\mathrm{C} 2-\mathrm{H} 2$ & 120.9 & $\mathrm{O} 51-\mathrm{N} 5-\mathrm{O} 52$ & $124.5(2)$ \\
\hline $\mathrm{C} 1-\mathrm{C} 2-\mathrm{H} 2$ & 120.9 & $\mathrm{O} 51-\mathrm{N} 5-\mathrm{C} 5$ & $118.01(19)$ \\
\hline $\mathrm{C} 2-\mathrm{C} 3-\mathrm{C} 4$ & $123.3(2)$ & $\mathrm{O} 52-\mathrm{N} 5-\mathrm{C} 5$ & $117.45(19)$ \\
\hline $\mathrm{C} 6-\mathrm{C} 1-\mathrm{C} 11-\mathrm{O} 1$ & $0.8(3)$ & $\mathrm{C} 4-\mathrm{C} 3-\mathrm{N} 3-\mathrm{O} 32$ & $-0.5(3)$ \\
\hline $\mathrm{C} 2-\mathrm{C} 1-\mathrm{C} 11-\mathrm{O} 1$ & $-179.2(2)$ & $\mathrm{C} 2-\mathrm{C} 3-\mathrm{C} 4-\mathrm{C} 5$ & $0.4(3)$ \\
\hline $\mathrm{C} 6-\mathrm{C} 1-\mathrm{C} 11-\mathrm{O} 11$ & $-178.01(19)$ & $\mathrm{N} 3-\mathrm{C} 3-\mathrm{C} 4-\mathrm{C} 5$ & $179.70(18)$ \\
\hline $\mathrm{C} 2-\mathrm{C} 1-\mathrm{C} 11-\mathrm{O} 11$ & $2.0(3)$ & $\mathrm{C} 2-\mathrm{C} 1-\mathrm{C} 6-\mathrm{C} 5$ & $0.0(3)$ \\
\hline $\mathrm{O} 1-\mathrm{C} 11-\mathrm{O} 11-\mathrm{C} 12$ & $1.0(3)$ & $\mathrm{C} 11-\mathrm{C} 1-\mathrm{C} 6-\mathrm{C} 5$ & $-179.97(19)$ \\
\hline $\mathrm{C} 1-\mathrm{C} 11-\mathrm{O} 11-\mathrm{C} 12$ & $179.74(19)$ & $\mathrm{C} 1-\mathrm{C} 6-\mathrm{C} 5-\mathrm{C} 4$ & $0.8(3)$ \\
\hline $\mathrm{C} 6-\mathrm{C} 1-\mathrm{C} 2-\mathrm{C} 3$ & $-0.6(3)$ & $\mathrm{C} 1-\mathrm{C} 6-\mathrm{C} 5-\mathrm{N} 5$ & $-179.33(19)$ \\
\hline
\end{tabular}




$\begin{array}{llll}\mathrm{C} 11-\mathrm{C} 1-\mathrm{C} 2-\mathrm{C} 3 & 179.4(2) & \mathrm{C} 3-\mathrm{C} 4-\mathrm{C} 5-\mathrm{C} 6 & -1.0(3) \\ \mathrm{C} 1-\mathrm{C} 2-\mathrm{C} 3-\mathrm{C} 4 & 0.4(3) & \mathrm{C} 3-\mathrm{C} 4-\mathrm{C} 5-\mathrm{N} 5 & 179.16(19) \\ \mathrm{C} 1-\mathrm{C} 2-\mathrm{C} 3-\mathrm{N} 3 & -178.96(19) & \mathrm{C} 6-\mathrm{C} 5-\mathrm{N} 5-\mathrm{O} 51 & -175.71(19) \\ \mathrm{C} 2-\mathrm{C} 3-\mathrm{N} 3-\mathrm{O} 31 & 0.2(3) & \mathrm{C} 4-\mathrm{C} 5-\mathrm{N} 5-\mathrm{O} 51 & 4.2(3) \\ \mathrm{C} 4-\mathrm{C} 3-\mathrm{N} 3-\mathrm{O} 31 & -179.2(2) & \mathrm{C} 6-\mathrm{C} 5-\mathrm{N} 5-\mathrm{O} 52 & 4.8(3) \\ \mathrm{C} 2-\mathrm{C} 3-\mathrm{N} 3-\mathrm{O} 32 & 178.86(19) & \mathrm{C} 4-\mathrm{C} 5-\mathrm{N} 5-\mathrm{O} 52 & -175.29(19)\end{array}$

Hydrogen-bond geometry $\left(\AA,{ }^{\circ}\right)$

\begin{tabular}{lllll}
\hline$D-\mathrm{H} \cdots A$ & $D-\mathrm{H}$ & $\mathrm{H} \cdots A$ & $D \cdots A$ & $D-\mathrm{H} \cdots A$ \\
\hline $\mathrm{C} 4-\mathrm{H} 4 \cdots \mathrm{O}^{\mathrm{i}}$ & 0.95 & 2.46 & $3.399(3)$ & 171 \\
\hline
\end{tabular}

Symmetry code: (i) $x+3 / 2,-y+3 / 2, z+1 / 2$.

(II) Isopropyl 3,5-dinitrobenzoate

Crystal data

$\mathrm{C}_{10} \mathrm{H}_{10} \mathrm{~N}_{2} \mathrm{O}_{6}$

$M_{r}=254.20$

Monoclinic, $P 2{ }_{1} / n$

Hall symbol: -P 2yn

$a=9.7037$ (3) $\AA$

$b=5.7152(2) \AA$

$c=20.4739(9) \AA$

$\beta=95.504(2)^{\circ}$

$V=1130.22(7) \AA^{3}$

$Z=4$

\section{Data collection}

Nonius KappaCCD diffractometer

Radiation source: Bruker-Nonius FR91 rotating anode

Graphite monochromator

Detector resolution: 9.091 pixels $\mathrm{mm}^{-1}$

$\varphi$ and $\omega$ scans

Absorption correction: multi-scan

(SADABS; Sheldrick, 2003)

\section{Refinement}

Refinement on $F^{2}$

Least-squares matrix: full

$R\left[F^{2}>2 \sigma\left(F^{2}\right)\right]=0.039$

$w R\left(F^{2}\right)=0.105$

$S=1.05$

2596 reflections

165 parameters

0 restraints

Primary atom site location: structure-invariant direct methods
$F(000)=528$

$D_{\mathrm{x}}=1.494 \mathrm{Mg} \mathrm{m}^{-3}$

Mo $K \alpha$ radiation, $\lambda=0.71073 \AA$

Cell parameters from 2596 reflections

$\theta=3.8-27.5^{\circ}$

$\mu=0.13 \mathrm{~mm}^{-1}$

$T=120 \mathrm{~K}$

Block, colourless

$0.42 \times 0.40 \times 0.38 \mathrm{~mm}$

$T_{\min }=0.944, T_{\max }=0.954$

10022 measured reflections

2596 independent reflections

2045 reflections with $I>2 \sigma(I)$

$R_{\text {int }}=0.031$

$\theta_{\max }=27.5^{\circ}, \theta_{\min }=3.8^{\circ}$

$h=-12 \rightarrow 12$

$k=-7 \rightarrow 7$

$l=-24 \rightarrow 26$

Secondary atom site location: difference Fourier map

Hydrogen site location: inferred from neighbouring sites

$\mathrm{H}$-atom parameters constrained

$w=1 /\left[\sigma^{2}\left(F_{\mathrm{o}}^{2}\right)+(0.0561 P)^{2}+0.2191 P\right]$

where $P=\left(F_{\mathrm{o}}^{2}+2 F_{\mathrm{c}}^{2}\right) / 3$

$(\Delta / \sigma)_{\max }=0.001$

$\Delta \rho_{\max }=0.30 \mathrm{e} \AA^{-3}$

$\Delta \rho_{\min }=-0.34$ e $\AA^{-3}$ 
Fractional atomic coordinates and isotropic or equivalent isotropic displacement parameters $\left(\AA^{2}\right)$

\begin{tabular}{|c|c|c|c|c|}
\hline & $x$ & $y$ & $z$ & $U_{\text {iso }} * / U_{\text {eq }}$ \\
\hline O1 & $0.27425(9)$ & $0.16199(16)$ & $0.63304(5)$ & $0.0258(2)$ \\
\hline O11 & $0.32924(8)$ & $0.47435(15)$ & $0.57331(4)$ & $0.0207(2)$ \\
\hline $\mathrm{O} 31$ & $0.63611(10)$ & $1.06799(16)$ & $0.67549(5)$ & $0.0288(2)$ \\
\hline $\mathrm{O} 32$ & $0.67594(9)$ & $1.08403(16)$ & $0.78148(5)$ & $0.0261(2)$ \\
\hline O51 & $0.52347(10)$ & $0.45491(17)$ & $0.91396(5)$ & $0.0314(3)$ \\
\hline $\mathrm{O} 52$ & $0.41232(9)$ & $0.16298(16)$ & $0.86658(5)$ & $0.0288(2)$ \\
\hline N3 & $0.62359(10)$ & $0.99248(18)$ & $0.73064(6)$ & $0.0208(2)$ \\
\hline N5 & $0.46624(10)$ & $0.35684(19)$ & $0.86574(5)$ & $0.0217(3)$ \\
\hline $\mathrm{C} 1$ & $0.39856(12)$ & $0.4773(2)$ & $0.68655(6)$ & $0.0178(3)$ \\
\hline $\mathrm{C} 2$ & $0.47145(11)$ & $0.6845(2)$ & $0.68063(6)$ & $0.0184(3)$ \\
\hline $\mathrm{C} 3$ & $0.54042(11)$ & $0.7796(2)$ & $0.73684(6)$ & $0.0180(3)$ \\
\hline $\mathrm{C} 4$ & $0.53871(11)$ & $0.6808(2)$ & $0.79833(6)$ & $0.0192(3)$ \\
\hline $\mathrm{C} 5$ & $0.46452(12)$ & $0.4756(2)$ & $0.80192(6)$ & $0.0182(3)$ \\
\hline C6 & $0.39433(11)$ & $0.3724(2)$ & $0.74748(6)$ & $0.0183(3)$ \\
\hline C11 & $0.32647(11)$ & $0.3529(2)$ & $0.62836(6)$ & $0.0184(3)$ \\
\hline $\mathrm{C} 12$ & $0.25439(13)$ & $0.3717(2)$ & $0.51385(6)$ & $0.0240(3)$ \\
\hline C13 & 0.21072 (19) & $0.5760(3)$ & $0.47026(8)$ & $0.0435(4)$ \\
\hline $\mathrm{C} 14$ & $0.35003(15)$ & 0.2029 & $0.48437(8)$ & $0.0357(4)$ \\
\hline $\mathrm{H} 2$ & 0.4739 & 0.7588 & 0.6392 & $0.022 *$ \\
\hline $\mathrm{H} 4$ & 0.5861 & 0.7504 & 0.8362 & $0.023 *$ \\
\hline H6 & 0.3439 & 0.2316 & 0.7517 & $0.022 *$ \\
\hline H12 & 0.1705 & 0.2865 & 0.5260 & $0.029 *$ \\
\hline H13A & 0.1584 & 0.6876 & 0.4946 & $0.065^{*}$ \\
\hline H13B & 0.1523 & 0.5203 & 0.4317 & $0.065 *$ \\
\hline $\mathrm{H} 13 \mathrm{C}$ & 0.2930 & 0.6533 & 0.4561 & $0.065 *$ \\
\hline H14A & 0.4342 & 0.2852 & 0.4745 & $0.053 *$ \\
\hline H14B & 0.3036 & 0.1374 & 0.4438 & $0.053 *$ \\
\hline $\mathrm{H} 14 \mathrm{C}$ & 0.3746 & 0.0762 & 0.5156 & $0.053 *$ \\
\hline
\end{tabular}

Atomic displacement parameters $\left(\AA^{2}\right)$

\begin{tabular}{lllllll}
\hline & $U^{11}$ & $U^{22}$ & $U^{33}$ & $U^{12}$ & $U^{13}$ & $U^{23}$ \\
\hline C1 & $0.0150(5)$ & $0.0180(6)$ & $0.0205(7)$ & $0.0023(5)$ & $0.0023(5)$ & $-0.0018(5)$ \\
C2 & $0.0164(5)$ & $0.0184(6)$ & $0.0211(6)$ & $0.0023(5)$ & $0.0045(5)$ & $0.0007(5)$ \\
C3 & $0.0145(5)$ & $0.0142(6)$ & $0.0257(7)$ & $0.0004(5)$ & $0.0042(5)$ & $-0.0016(5)$ \\
N3 & $0.0171(5)$ & $0.0175(5)$ & $0.0283(6)$ & $-0.0001(4)$ & $0.0043(4)$ & $-0.0031(4)$ \\
O31 & $0.0308(5)$ & $0.0251(5)$ & $0.0311(6)$ & $-0.0060(4)$ & $0.0072(4)$ & $0.0035(4)$ \\
O32 & $0.0231(4)$ & $0.0227(5)$ & $0.0325(6)$ & $-0.0042(4)$ & $0.0028(4)$ & $-0.0069(4)$ \\
C4 & $0.0153(5)$ & $0.0197(6)$ & $0.0225(7)$ & $0.0017(5)$ & $0.0015(5)$ & $-0.0039(5)$ \\
C5 & $0.0160(5)$ & $0.0191(6)$ & $0.0197(7)$ & $0.0035(5)$ & $0.0031(4)$ & $0.0008(5)$ \\
N5 & $0.0179(5)$ & $0.0251(6)$ & $0.0219(6)$ & $0.0005(4)$ & $0.0007(4)$ & $0.0020(5)$ \\
O51 & $0.0359(5)$ & $0.0350(6)$ & $0.0216(5)$ & $-0.0047(4)$ & $-0.0061(4)$ & $0.0003(4)$ \\
O52 & $0.0301(5)$ & $0.0267(5)$ & $0.0296(6)$ & $-0.0074(4)$ & $0.0029(4)$ & $0.0062(4)$ \\
C6 & $0.0142(5)$ & $0.0165(6)$ & $0.0247(7)$ & $0.0008(5)$ & $0.0038(5)$ & $0.0002(5)$ \\
C11 & $0.0147(5)$ & $0.0192(6)$ & $0.0214(7)$ & $0.0002(5)$ & $0.0026(5)$ & $-0.0004(5)$
\end{tabular}




\begin{tabular}{lllllll}
$\mathrm{O} 1$ & $0.0279(5)$ & $0.0233(5)$ & $0.0257(5)$ & $-0.0080(4)$ & $0.0008(4)$ & $0.0003(4)$ \\
$\mathrm{O} 11$ & $0.0235(4)$ & $0.0212(5)$ & $0.0170(5)$ & $-0.0038(4)$ & $0.0001(3)$ & $-0.0005(3)$ \\
$\mathrm{C} 12$ & $0.0267(6)$ & $0.0248(7)$ & $0.0193(7)$ & $-0.0063(5)$ & $-0.0032(5)$ & $-0.0014(5)$ \\
$\mathrm{C} 13$ & $0.0634(11)$ & $0.0327(8)$ & $0.0302(9)$ & $-0.0079(8)$ & $-0.0173(8)$ & $0.0055(7)$ \\
$\mathrm{C} 14$ & $0.0374(8)$ & $0.0390(8)$ & $0.0313(8)$ & $-0.0078(7)$ & $0.0065(6)$ & $-0.0141(7)$ \\
\hline
\end{tabular}

Geometric parameters $\left(\hat{A},{ }^{\circ}\right)$

\begin{tabular}{|c|c|c|c|}
\hline $\mathrm{C} 1-\mathrm{C} 6$ & $1.3883(17)$ & $\mathrm{N} 5-\mathrm{O} 52$ & $1.2261(14)$ \\
\hline $\mathrm{C} 1-\mathrm{C} 2$ & $1.3907(17)$ & C6- $\mathrm{H} 6$ & 0.95 \\
\hline $\mathrm{C} 1-\mathrm{C} 11$ & $1.5014(17)$ & $\mathrm{C} 11-\mathrm{O} 1$ & $1.2109(15)$ \\
\hline $\mathrm{C} 2-\mathrm{C} 3$ & $1.3856(17)$ & $\mathrm{C} 11-\mathrm{O} 11$ & $1.3260(15)$ \\
\hline $\mathrm{C} 2-\mathrm{H} 2$ & 0.95 & $\mathrm{O} 11-\mathrm{C} 12$ & $1.4778(15)$ \\
\hline $\mathrm{C} 3-\mathrm{C} 4$ & $1.3813(17)$ & $\mathrm{C} 12-\mathrm{C} 14$ & $1.505(2)$ \\
\hline $\mathrm{C} 3-\mathrm{N} 3$ & $1.4721(16)$ & $\mathrm{C} 12-\mathrm{C} 13$ & $1.5058(19)$ \\
\hline $\mathrm{N} 3-\mathrm{O} 31$ & $1.2257(14)$ & $\mathrm{C} 12-\mathrm{H} 12$ & 1.00 \\
\hline $\mathrm{N} 3-\mathrm{O} 32$ & $1.2303(14)$ & $\mathrm{C} 13-\mathrm{H} 13 \mathrm{~A}$ & 0.98 \\
\hline $\mathrm{C} 4-\mathrm{C} 5$ & $1.3819(17)$ & C13-H13B & 0.98 \\
\hline $\mathrm{C} 4-\mathrm{H} 4$ & 0.95 & $\mathrm{C} 13-\mathrm{H} 13 \mathrm{C}$ & 0.98 \\
\hline $\mathrm{C} 5-\mathrm{C} 6$ & $1.3815(17)$ & $\mathrm{C} 14-\mathrm{H} 14 \mathrm{~A}$ & 0.98 \\
\hline $\mathrm{C} 5-\mathrm{N} 5$ & $1.4710(16)$ & $\mathrm{C} 14-\mathrm{H} 14 \mathrm{~B}$ & 0.98 \\
\hline $\mathrm{N} 5-\mathrm{O} 51$ & $1.2216(14)$ & $\mathrm{C} 14-\mathrm{H} 14 \mathrm{C}$ & 0.98 \\
\hline $\mathrm{C} 6-\mathrm{C} 1-\mathrm{C} 2$ & $120.34(11)$ & $\mathrm{C} 1-\mathrm{C} 6-\mathrm{H} 6$ & 120.5 \\
\hline $\mathrm{C} 6-\mathrm{C} 1-\mathrm{C} 11$ & $117.19(11)$ & $\mathrm{O} 1-\mathrm{C} 11-\mathrm{O} 11$ & $125.56(11)$ \\
\hline $\mathrm{C} 2-\mathrm{C} 1-\mathrm{C} 11$ & $122.43(11)$ & $\mathrm{O} 1-\mathrm{C} 11-\mathrm{C} 1$ & $122.05(11)$ \\
\hline $\mathrm{C} 3-\mathrm{C} 2-\mathrm{C} 1$ & $118.09(12)$ & $\mathrm{O} 11-\mathrm{C} 11-\mathrm{C} 1$ & $112.38(10)$ \\
\hline $\mathrm{C} 3-\mathrm{C} 2-\mathrm{H} 2$ & 121.0 & $\mathrm{C} 11-\mathrm{O} 11-\mathrm{C} 12$ & $116.36(10)$ \\
\hline $\mathrm{C} 1-\mathrm{C} 2-\mathrm{H} 2$ & 121.0 & $\mathrm{O} 11-\mathrm{C} 12-\mathrm{C} 14$ & $108.16(11)$ \\
\hline $\mathrm{C} 4-\mathrm{C} 3-\mathrm{C} 2$ & $123.34(11)$ & $\mathrm{O} 11-\mathrm{C} 12-\mathrm{C} 13$ & $105.57(11)$ \\
\hline $\mathrm{C} 4-\mathrm{C} 3-\mathrm{N} 3$ & $118.10(11)$ & $\mathrm{C} 14-\mathrm{C} 12-\mathrm{C} 13$ & $114.13(13)$ \\
\hline $\mathrm{C} 2-\mathrm{C} 3-\mathrm{N} 3$ & $118.53(11)$ & $\mathrm{O} 11-\mathrm{C} 12-\mathrm{H} 12$ & 109.6 \\
\hline $\mathrm{O} 31-\mathrm{N} 3-\mathrm{O} 32$ & $124.07(11)$ & $\mathrm{C} 14-\mathrm{C} 12-\mathrm{H} 12$ & 109.6 \\
\hline $\mathrm{O} 31-\mathrm{N} 3-\mathrm{C} 3$ & $118.30(10)$ & $\mathrm{C} 13-\mathrm{C} 12-\mathrm{H} 12$ & 109.6 \\
\hline $\mathrm{O} 32-\mathrm{N} 3-\mathrm{C} 3$ & $117.63(11)$ & $\mathrm{C} 12-\mathrm{C} 13-\mathrm{H} 13 \mathrm{~A}$ & 109.5 \\
\hline $\mathrm{C} 3-\mathrm{C} 4-\mathrm{C} 5$ & $116.57(11)$ & $\mathrm{C} 12-\mathrm{C} 13-\mathrm{H} 13 \mathrm{~B}$ & 109.5 \\
\hline $\mathrm{C} 3-\mathrm{C} 4-\mathrm{H} 4$ & 121.7 & $\mathrm{H} 13 \mathrm{~A}-\mathrm{C} 13-\mathrm{H} 13 \mathrm{~B}$ & 109.5 \\
\hline $\mathrm{C} 5-\mathrm{C} 4-\mathrm{H} 4$ & 121.7 & $\mathrm{C} 12-\mathrm{C} 13-\mathrm{H} 13 \mathrm{C}$ & 109.5 \\
\hline $\mathrm{C} 6-\mathrm{C} 5-\mathrm{C} 4$ & $122.58(12)$ & $\mathrm{H} 13 \mathrm{~A}-\mathrm{C} 13-\mathrm{H} 13 \mathrm{C}$ & 109.5 \\
\hline $\mathrm{C} 6-\mathrm{C} 5-\mathrm{N} 5$ & $118.84(11)$ & $\mathrm{H} 13 \mathrm{~B}-\mathrm{C} 13-\mathrm{H} 13 \mathrm{C}$ & 109.5 \\
\hline $\mathrm{C} 4-\mathrm{C} 5-\mathrm{N} 5$ & $118.51(11)$ & $\mathrm{C} 12-\mathrm{C} 14-\mathrm{H} 14 \mathrm{~A}$ & 109.5 \\
\hline $\mathrm{O} 51-\mathrm{N} 5-\mathrm{O} 52$ & $124.39(11)$ & $\mathrm{C} 12-\mathrm{C} 14-\mathrm{H} 14 \mathrm{~B}$ & 109.5 \\
\hline $\mathrm{O} 51-\mathrm{N} 5-\mathrm{C} 5$ & $118.15(11)$ & $\mathrm{H} 14 \mathrm{~A}-\mathrm{C} 14-\mathrm{H} 14 \mathrm{~B}$ & 109.5 \\
\hline $\mathrm{O} 52-\mathrm{N} 5-\mathrm{C} 5$ & $117.45(10)$ & $\mathrm{C} 12-\mathrm{C} 14-\mathrm{H} 14 \mathrm{C}$ & 109.5 \\
\hline $\mathrm{C} 5-\mathrm{C} 6-\mathrm{C} 1$ & $119.07(12)$ & $\mathrm{H} 14 \mathrm{~A}-\mathrm{C} 14-\mathrm{H} 14 \mathrm{C}$ & 109.5 \\
\hline $\mathrm{C} 5-\mathrm{C} 6-\mathrm{H} 6$ & 120.5 & $\mathrm{H} 14 \mathrm{~B}-\mathrm{C} 14-\mathrm{H} 14 \mathrm{C}$ & 109.5 \\
\hline $\mathrm{C} 6-\mathrm{C} 1-\mathrm{C} 2-\mathrm{C} 3$ & $0.88(17)$ & $\mathrm{C} 6-\mathrm{C} 5-\mathrm{N} 5-\mathrm{O} 52$ & $4.66(16)$ \\
\hline
\end{tabular}




$\begin{array}{ll}\mathrm{C} 11-\mathrm{C} 1-\mathrm{C} 2-\mathrm{C} 3 & -176.74(10) \\ \mathrm{C} 1-\mathrm{C} 2-\mathrm{C} 3-\mathrm{C} 4 & -0.94(17) \\ \mathrm{C} 1-\mathrm{C} 2-\mathrm{C} 3-\mathrm{N} 3 & 176.99(10) \\ \mathrm{C} 4-\mathrm{C} 3-\mathrm{N} 3-\mathrm{O} 31 & 173.16(10) \\ \mathrm{C} 2-\mathrm{C} 3-\mathrm{N} 3-\mathrm{O} 31 & -4.88(16) \\ \mathrm{C} 4-\mathrm{C} 3-\mathrm{N} 3-\mathrm{O} 32 & -7.08(15) \\ \mathrm{C} 2-\mathrm{C} 3-\mathrm{N} 3-\mathrm{O} 32 & 174.88(10) \\ \mathrm{C} 2-\mathrm{C} 3-\mathrm{C} 4-\mathrm{C} 5 & 0.67(17) \\ \mathrm{N} 3-\mathrm{C} 3-\mathrm{C} 4-\mathrm{C} 5 & -177.27(10) \\ \mathrm{C} 3-\mathrm{C} 4-\mathrm{C} 5-\mathrm{C} 6 & -0.34(17) \\ \mathrm{C} 3-\mathrm{C} 4-\mathrm{C} 5-\mathrm{N} 5 & 176.57(10) \\ \mathrm{C} 6-\mathrm{C} 5-\mathrm{N} 5-\mathrm{O} 51 & -176.84(11) \\ \mathrm{C} 4-\mathrm{C} 5-\mathrm{N} 5-\mathrm{O} 51 & 6.13(16)\end{array}$

$\mathrm{C} 4-\mathrm{C} 5-\mathrm{N} 5-\mathrm{O} 52$
$\mathrm{C} 4-\mathrm{C} 5-\mathrm{C} 6-\mathrm{C} 1$
$\mathrm{~N} 5-\mathrm{C} 5-\mathrm{C} 6-\mathrm{C} 1$
$\mathrm{C} 2-\mathrm{C} 1-\mathrm{C} 6-\mathrm{C} 5$
$\mathrm{C} 11-\mathrm{C} 1-\mathrm{C} 6-\mathrm{C} 5$
$\mathrm{C} 6-\mathrm{C} 1-\mathrm{C} 11-\mathrm{O} 1$
$\mathrm{C} 2-\mathrm{C} 1-\mathrm{C} 11-\mathrm{O} 1$
$\mathrm{C} 6-\mathrm{C} 1-\mathrm{C} 11-\mathrm{O} 11$
$\mathrm{C} 2-\mathrm{C} 1-\mathrm{C} 11-\mathrm{O} 11$
$\mathrm{O} 1-\mathrm{C} 11-\mathrm{O} 11-\mathrm{C} 12$
$\mathrm{C} 1-\mathrm{C} 11-\mathrm{O} 11-\mathrm{C} 12$
$\mathrm{C} 11-\mathrm{O} 11-\mathrm{C} 12-\mathrm{C} 14$
$\mathrm{C} 11-\mathrm{O} 11-\mathrm{C} 12-\mathrm{C} 13$

$-172.37(11)$

$0.32(18)$

$-176.58(10)$

-0.59 (17)

$177.15(10)$

$-4.72(17)$

$172.98(11)$

$176.07(10)$

$-6.24(16)$

$3.94(17)$

$-176.88(9)$

$-85.33(13)$

$152.12(12)$

(III) Benzyl 3,5-dinitrobenzoate?

Crystal data

$\mathrm{C}_{14} \mathrm{H}_{10} \mathrm{~N}_{2} \mathrm{O}_{6}$

$M_{r}=302.24$

Tetragonal, $P 4_{2} / n$

Hall symbol: -P $4 \mathrm{bc}$

$a=20.8531(5) \AA$

$c=6.0377(2) \AA$

$V=2625.50(12) \AA^{3}$

$Z=8$

$F(000)=1248$

Data collection

Nonius KappaCCD diffractometer

Radiation source: Bruker-Nonius FR91 rotating anode

Graphite monochromator

Detector resolution: 9.091 pixels $\mathrm{mm}^{-1}$

$\varphi$ and $\omega$ scans

Absorption correction: multi-scan

(SADABS; Sheldrick, 2003)

Refinement

Refinement on $F^{2}$

Least-squares matrix: full

$R\left[F^{2}>2 \sigma\left(F^{2}\right)\right]=0.043$

$w R\left(F^{2}\right)=0.103$

$S=1.05$

3000 reflections

199 parameters

0 restraints

Primary atom site location: structure-invariant direct methods
$D_{\mathrm{x}}=1.529 \mathrm{Mg} \mathrm{m}^{-3}$

Mo $K \alpha$ radiation, $\lambda=0.71073 \AA$

Cell parameters from 3000 reflections

$\theta=3.1-27.5^{\circ}$

$\mu=0.12 \mathrm{~mm}^{-1}$

$T=120 \mathrm{~K}$

Needle, colourless

$0.22 \times 0.06 \times 0.04 \mathrm{~mm}$

$T_{\min }=0.978, T_{\max }=0.995$

18873 measured reflections

3000 independent reflections

2413 reflections with $I>2 \sigma(I)$

$R_{\text {int }}=0.053$

$\theta_{\max }=27.5^{\circ}, \theta_{\min }=3.1^{\circ}$

$h=-27 \rightarrow 26$

$k=-16 \rightarrow 27$

$l=-7 \rightarrow 7$

Secondary atom site location: difference Fourier map

Hydrogen site location: inferred from neighbouring sites

$\mathrm{H}$-atom parameters constrained

$w=1 /\left[\sigma^{2}\left(F_{\mathrm{o}}^{2}\right)+(0.0355 P)^{2}+1.5651 P\right]$

where $P=\left(F_{\mathrm{o}}{ }^{2}+2 F_{\mathrm{c}}{ }^{2}\right) / 3$

$(\Delta / \sigma)_{\max }=0.001$

$\Delta \rho_{\max }=0.24 \mathrm{e} \AA^{-3}$

$\Delta \rho_{\min }=-0.23$ e $\AA^{-3}$ 
Fractional atomic coordinates and isotropic or equivalent isotropic displacement parameters $\left(\AA^{2}\right)$

\begin{tabular}{|c|c|c|c|c|}
\hline & $x$ & $y$ & $z$ & $U_{\text {iso }} * / U_{\text {eq }}$ \\
\hline $\mathrm{O} 1$ & $0.49125(5)$ & $0.44104(6)$ & $0.1932(2)$ & $0.0254(3)$ \\
\hline O11 & $0.52732(5)$ & $0.40805(5)$ & $0.52649(18)$ & $0.0211(3)$ \\
\hline $\mathrm{O} 31$ & $0.74509(6)$ & $0.32867(7)$ & $0.5879(2)$ & $0.0376(3)$ \\
\hline $\mathrm{O} 32$ & $0.81810(5)$ & $0.37069(6)$ & $0.3788(2)$ & $0.0284(3)$ \\
\hline O51 & $0.75704(5)$ & $0.47883(6)$ & $-0.2874(2)$ & $0.0273(3)$ \\
\hline O52 & $0.66120(6)$ & $0.51811(6)$ & $-0.3183(2)$ & $0.0287(3)$ \\
\hline N3 & $0.76221(6)$ & $0.36163(7)$ & $0.4312(2)$ & $0.0226(3)$ \\
\hline N5 & $0.70199(6)$ & $0.48641(6)$ & $-0.2208(2)$ & $0.0206(3)$ \\
\hline $\mathrm{C} 1$ & $0.60325(7)$ & $0.42401(7)$ & $0.2460(3)$ & $0.0172(3)$ \\
\hline $\mathrm{C} 2$ & $0.64954(7)$ & $0.39295(7)$ & $0.3724(3)$ & $0.0181(3)$ \\
\hline $\mathrm{C} 3$ & $0.71241(7)$ & $0.39371(7)$ & $0.2979(3)$ & $0.0188(3)$ \\
\hline $\mathrm{C} 4$ & $0.73094(7)$ & $0.42425(7)$ & $0.1063(3)$ & $0.0185(3)$ \\
\hline $\mathrm{C} 5$ & $0.68335(7)$ & $0.45441(7)$ & $-0.0135(3)$ & $0.0175(3)$ \\
\hline C6 & $0.61973(7)$ & $0.45520(7)$ & 0.0503 & $0.0180(3)$ \\
\hline $\mathrm{C} 11$ & $0.53428(7)$ & $0.42524(7)$ & $0.3149(3)$ & $0.0181(3)$ \\
\hline $\mathrm{C} 12$ & $0.46199(7)$ & $0.41048(8)$ & $0.6171(3)$ & $0.0209(3)$ \\
\hline $\mathrm{C} 21$ & $0.42942(7)$ & $0.34636(7)$ & $0.5979(3)$ & $0.0192(3)$ \\
\hline $\mathrm{C} 22$ & $0.43489(8)$ & $0.30202(8)$ & 0.7690 & $0.0242(4)$ \\
\hline $\mathrm{C} 23$ & $0.40476(9)$ & $0.24289(9)$ & $0.7537(3)$ & $0.0296(4)$ \\
\hline $\mathrm{C} 24$ & $0.36916(8)$ & $0.22777(8)$ & 0.5671 & $0.0293(4)$ \\
\hline $\mathrm{C} 25$ & $0.36404(8)$ & $0.27124(8)$ & 0.3950 & $0.0270(4)$ \\
\hline $\mathrm{C} 26$ & $0.39397(8)$ & $0.33054(8)$ & $0.4104(3)$ & $0.0219(4)$ \\
\hline $\mathrm{H} 2$ & 0.6384 & 0.3718 & 0.5063 & $0.022 *$ \\
\hline $\mathrm{H} 4$ & 0.7744 & 0.4246 & 0.0588 & $0.022 *$ \\
\hline H6 & 0.5882 & 0.4764 & -0.0367 & $0.022 *$ \\
\hline $\mathrm{H} 12 \mathrm{~A}$ & 0.4367 & 0.4431 & 0.5359 & $0.025^{*}$ \\
\hline H12B & 0.4638 & 0.4234 & 0.7748 & $0.025 *$ \\
\hline $\mathrm{H} 22$ & 0.4594 & 0.3123 & 0.8968 & $0.029 *$ \\
\hline $\mathrm{H} 23$ & 0.4085 & 0.2128 & 0.8711 & $0.036^{*}$ \\
\hline $\mathrm{H} 24$ & 0.3482 & 0.1874 & 0.5573 & $0.035 *$ \\
\hline $\mathrm{H} 25$ & 0.3401 & 0.2605 & 0.2664 & $0.032 *$ \\
\hline $\mathrm{H} 26$ & 0.3902 & 0.3604 & 0.2924 & $0.026^{*}$ \\
\hline
\end{tabular}

Atomic displacement parameters $\left(\AA^{2}\right)$

\begin{tabular}{lllllll}
\hline & $U^{11}$ & $U^{22}$ & $U^{33}$ & $U^{12}$ & $U^{13}$ & $U^{23}$ \\
\hline C1 & $0.0169(7)$ & $0.0146(7)$ & $0.0201(8)$ & $-0.0015(6)$ & $-0.0002(6)$ & $-0.0025(6)$ \\
C2 & $0.0196(8)$ & $0.0159(7)$ & $0.0187(8)$ & $-0.0025(6)$ & $-0.0007(6)$ & $-0.0005(6)$ \\
C3 & $0.0183(8)$ & $0.0171(8)$ & $0.0210(8)$ & $0.0011(6)$ & $-0.0031(6)$ & $-0.0015(6)$ \\
N3 & $0.0191(7)$ & $0.0253(7)$ & $0.0235(7)$ & $0.0027(5)$ & $-0.0016(6)$ & $0.0009(6)$ \\
O32 & $0.0155(6)$ & $0.0409(7)$ & $0.0287(7)$ & $0.0048(5)$ & $-0.0011(5)$ & $0.0001(6)$ \\
O31 & $0.0285(7)$ & $0.0473(8)$ & $0.0369(8)$ & $0.0044(6)$ & $-0.0004(6)$ & $0.0228(7)$ \\
C4 & $0.0164(7)$ & $0.0173(8)$ & $0.0218(8)$ & $-0.0009(6)$ & $0.0004(6)$ & $-0.0026(6)$ \\
C5 & $0.0207(8)$ & $0.0137(7)$ & $0.0182(8)$ & $-0.0025(6)$ & $0.0013(6)$ & $-0.0013(6)$ \\
N5 & $0.0222(7)$ & $0.0188(7)$ & $0.0206(7)$ & $-0.0022(5)$ & $0.0009(6)$ & $0.0004(6)$
\end{tabular}




$\begin{array}{lllllll}\text { O52 } & 0.0285(7) & 0.0305(7) & 0.0271(7) & 0.0026(5) & -0.0010(5) & 0.0108(5) \\ \text { O51 } & 0.0220(6) & 0.0325(7) & 0.0275(7) & -0.0003(5) & 0.0074(5) & 0.0030(5) \\ \text { C6 } & 0.0185(8) & 0.0151(7) & 0.0206(8) & -0.0009(6) & -0.0025(6) & -0.0013(6) \\ \text { C11 } & 0.0187(8) & 0.0152(7) & 0.0204(8) & -0.0015(6) & -0.0013(6) & 0.0012(6) \\ \text { O1 } & 0.0184(6) & 0.0317(7) & 0.0261(6) & 0.0013(5) & -0.0020(5) & 0.0065(5) \\ \text { O11 } & 0.0150(5) & 0.0283(6) & 0.0199(6) & -0.0014(4) & 0.0014(5) & 0.0028(5) \\ \text { C12 } & 0.0160(8) & 0.0260(8) & 0.0207(8) & 0.0001(6) & 0.0035(6) & -0.0016(7) \\ \text { C21 } & 0.0147(7) & 0.0211(8) & 0.0218(8) & 0.0026(6) & 0.0037(6) & 0.0004(6) \\ \text { C22 } & 0.0210(8) & 0.0288(9) & 0.0229(9) & 0.0031(7) & 0.0005(7) & 0.0040(7) \\ \text { C23 } & 0.0294(10) & 0.0261(9) & 0.0334(10) & 0.0044(7) & 0.0049(8) & 0.0102(8) \\ \text { C24 } & 0.0271(9) & 0.0192(8) & 0.0414(11) & -0.0025(7) & 0.0035(8) & 0.0006(8) \\ \text { C25 } & 0.0250(9) & 0.0243(9) & 0.0318(10) & -0.0023(7) & -0.0032(7) & -0.0018(7) \\ \text { C26 } & 0.0201(8) & 0.0227(8) & 0.0230(9) & 0.0017(6) & -0.0011(7) & 0.0031(7) \\ & & & & & \end{array}$

Geometric parameters $\left(\AA,{ }^{\circ}\right)$

\begin{tabular}{|c|c|c|c|}
\hline $\mathrm{C} 1-\mathrm{C} 2$ & $1.391(2)$ & $\mathrm{C} 11-\mathrm{O} 11$ & $1.3350(19)$ \\
\hline $\mathrm{C} 1-\mathrm{C} 6$ & $1.392(2)$ & $\mathrm{O} 11-\mathrm{C} 12$ & $1.4690(18)$ \\
\hline $\mathrm{C} 1-\mathrm{C} 11$ & $1.497(2)$ & $\mathrm{C} 12-\mathrm{C} 21$ & $1.504(2)$ \\
\hline $\mathrm{C} 2-\mathrm{C} 3$ & $1.386(2)$ & $\mathrm{C} 12-\mathrm{H} 12 \mathrm{~A}$ & 0.99 \\
\hline $\mathrm{C} 2-\mathrm{H} 2$ & 0.95 & $\mathrm{C} 12-\mathrm{H} 12 \mathrm{~B}$ & 0.99 \\
\hline $\mathrm{C} 3-\mathrm{C} 4$ & $1.376(2)$ & $\mathrm{C} 21-\mathrm{C} 22$ & $1.391(2)$ \\
\hline $\mathrm{C} 3-\mathrm{N} 3$ & $1.474(2)$ & $\mathrm{C} 21-\mathrm{C} 26$ & $1.392(2)$ \\
\hline $\mathrm{N} 3-\mathrm{O} 32$ & $1.2223(17)$ & $\mathrm{C} 22-\mathrm{C} 23$ & $1.387(2)$ \\
\hline N3-O31 & $1.2224(18)$ & $\mathrm{C} 22-\mathrm{H} 22$ & 0.95 \\
\hline $\mathrm{C} 4-\mathrm{C} 5$ & $1.380(2)$ & $\mathrm{C} 23-\mathrm{C} 24$ & $1.386(3)$ \\
\hline $\mathrm{C} 4-\mathrm{H} 4$ & 0.95 & $\mathrm{C} 23-\mathrm{H} 23$ & 0.95 \\
\hline $\mathrm{C} 5-\mathrm{C} 6$ & $1.381(2)$ & $\mathrm{C} 24-\mathrm{C} 25$ & $1.383(3)$ \\
\hline $\mathrm{C} 5-\mathrm{N} 5$ & $1.471(2)$ & $\mathrm{C} 24-\mathrm{H} 24$ & 0.95 \\
\hline $\mathrm{N} 5-\mathrm{O} 51$ & $1.2263(17)$ & $\mathrm{C} 25-\mathrm{C} 26$ & $1.388(2)$ \\
\hline $\mathrm{N} 5-\mathrm{O} 52$ & $1.2275(17)$ & $\mathrm{C} 25-\mathrm{H} 25$ & 0.95 \\
\hline C6- & 0.95 & $\mathrm{C} 26-\mathrm{H} 26$ & 0.95 \\
\hline $\mathrm{C} 11-\mathrm{O} 1$ & $1.2053(19)$ & & \\
\hline $\mathrm{C} 2-\mathrm{C} 1-\mathrm{C} 6$ & $120.82(14)$ & $\mathrm{O} 11-\mathrm{C} 11-\mathrm{C} 1$ & $111.45(13)$ \\
\hline $\mathrm{C} 2-\mathrm{C} 1-\mathrm{C} 11$ & $121.48(14)$ & $\mathrm{C} 11-\mathrm{O} 11-\mathrm{C} 12$ & $116.61(12)$ \\
\hline $\mathrm{C} 6-\mathrm{C} 1-\mathrm{C} 11$ & $117.70(14)$ & $\mathrm{O} 11-\mathrm{C} 12-\mathrm{C} 21$ & $111.06(12)$ \\
\hline $\mathrm{C} 3-\mathrm{C} 2-\mathrm{C} 1$ & $118.23(15)$ & $\mathrm{O} 11-\mathrm{C} 12-\mathrm{H} 12 \mathrm{~A}$ & 109.4 \\
\hline $\mathrm{C} 3-\mathrm{C} 2-\mathrm{H} 2$ & 120.9 & $\mathrm{C} 21-\mathrm{C} 12-\mathrm{H} 12 \mathrm{~A}$ & 109.4 \\
\hline $\mathrm{C} 1-\mathrm{C} 2-\mathrm{H} 2$ & 120.9 & $\mathrm{O} 11-\mathrm{C} 12-\mathrm{H} 12 \mathrm{~B}$ & 109.4 \\
\hline $\mathrm{C} 4-\mathrm{C} 3-\mathrm{C} 2$ & $122.94(15)$ & $\mathrm{C} 21-\mathrm{C} 12-\mathrm{H} 12 \mathrm{~B}$ & 109.4 \\
\hline $\mathrm{C} 4-\mathrm{C} 3-\mathrm{N} 3$ & $118.11(14)$ & $\mathrm{H} 12 \mathrm{~A}-\mathrm{C} 12-\mathrm{H} 12 \mathrm{~B}$ & 108.0 \\
\hline $\mathrm{C} 2-\mathrm{C} 3-\mathrm{N} 3$ & $118.93(14)$ & $\mathrm{C} 22-\mathrm{C} 21-\mathrm{C} 26$ & $119.35(15)$ \\
\hline $\mathrm{O} 32-\mathrm{N} 3-\mathrm{O} 31$ & $124.45(14)$ & $\mathrm{C} 22-\mathrm{C} 21-\mathrm{C} 12$ & $119.77(15)$ \\
\hline $\mathrm{O} 32-\mathrm{N} 3-\mathrm{C} 3$ & $117.39(13)$ & $\mathrm{C} 26-\mathrm{C} 21-\mathrm{C} 12$ & $120.88(14)$ \\
\hline $\mathrm{O} 31-\mathrm{N} 3-\mathrm{C} 3$ & $118.15(13)$ & $\mathrm{C} 23-\mathrm{C} 22-\mathrm{C} 21$ & $120.28(16)$ \\
\hline $\mathrm{C} 3-\mathrm{C} 4-\mathrm{C} 5$ & $116.73(14)$ & $\mathrm{C} 23-\mathrm{C} 22-\mathrm{H} 22$ & 119.9 \\
\hline $\mathrm{C} 3-\mathrm{C} 4-\mathrm{H} 4$ & 121.6 & $\mathrm{C} 21-\mathrm{C} 22-\mathrm{H} 22$ & 119.9 \\
\hline
\end{tabular}




\begin{tabular}{|c|c|c|c|}
\hline $\mathrm{C} 5-\mathrm{C} 4-\mathrm{H} 4$ & 121.6 & $\mathrm{C} 24-\mathrm{C} 23-\mathrm{C} 22$ & $119.95(16)$ \\
\hline $\mathrm{C} 4-\mathrm{C} 5-\mathrm{C} 6$ & $123.37(15)$ & $\mathrm{C} 24-\mathrm{C} 23-\mathrm{H} 23$ & 120.0 \\
\hline $\mathrm{C} 4-\mathrm{C} 5-\mathrm{N} 5$ & $117.57(14)$ & $\mathrm{C} 22-\mathrm{C} 23-\mathrm{H} 23$ & 120.0 \\
\hline $\mathrm{C} 6-\mathrm{C} 5-\mathrm{N} 5$ & $119.06(14)$ & $\mathrm{C} 25-\mathrm{C} 24-\mathrm{C} 23$ & $120.20(16)$ \\
\hline $\mathrm{O} 51-\mathrm{N} 5-\mathrm{O} 52$ & $124.14(14)$ & $\mathrm{C} 25-\mathrm{C} 24-\mathrm{H} 24$ & 119.9 \\
\hline $\mathrm{O} 51-\mathrm{N} 5-\mathrm{C} 5$ & $117.88(13)$ & $\mathrm{C} 23-\mathrm{C} 24-\mathrm{H} 24$ & 119.9 \\
\hline $\mathrm{O} 52-\mathrm{N} 5-\mathrm{C} 5$ & $117.96(13)$ & $\mathrm{C} 24-\mathrm{C} 25-\mathrm{C} 26$ & $119.93(17)$ \\
\hline $\mathrm{C} 5-\mathrm{C} 6-\mathrm{C} 1$ & $117.92(14)$ & $\mathrm{C} 24-\mathrm{C} 25-\mathrm{H} 25$ & 120.0 \\
\hline $\mathrm{C} 5-\mathrm{C} 6-\mathrm{H} 6$ & 121.0 & $\mathrm{C} 26-\mathrm{C} 25-\mathrm{H} 25$ & 120.0 \\
\hline $\mathrm{C} 1-\mathrm{C} 6-\mathrm{H} 6$ & 121.0 & $\mathrm{C} 25-\mathrm{C} 26-\mathrm{C} 21$ & $120.29(16)$ \\
\hline $\mathrm{O} 1-\mathrm{C} 11-\mathrm{O} 11$ & $125.14(15)$ & $\mathrm{C} 25-\mathrm{C} 26-\mathrm{H} 26$ & 119.9 \\
\hline $\mathrm{O} 1-\mathrm{C} 11-\mathrm{C} 1$ & $123.40(15)$ & $\mathrm{C} 21-\mathrm{C} 26-\mathrm{H} 26$ & 119.9 \\
\hline $\mathrm{C} 6-\mathrm{C} 1-\mathrm{C} 2-\mathrm{C} 3$ & $-0.2(2)$ & $\mathrm{C} 11-\mathrm{C} 1-\mathrm{C} 6-\mathrm{C} 5$ & $179.75(13)$ \\
\hline $\mathrm{C} 11-\mathrm{C} 1-\mathrm{C} 2-\mathrm{C} 3$ & $179.86(14)$ & $\mathrm{C} 2-\mathrm{C} 1-\mathrm{C} 11-\mathrm{O} 1$ & $-166.04(15)$ \\
\hline $\mathrm{C} 1-\mathrm{C} 2-\mathrm{C} 3-\mathrm{C} 4$ & $0.6(2)$ & $\mathrm{C} 6-\mathrm{C} 1-\mathrm{C} 11-\mathrm{O} 1$ & $14.0(2)$ \\
\hline $\mathrm{C} 1-\mathrm{C} 2-\mathrm{C} 3-\mathrm{N} 3$ & $179.32(13)$ & $\mathrm{C} 2-\mathrm{C} 1-\mathrm{C} 11-\mathrm{O} 11$ & $15.2(2)$ \\
\hline $\mathrm{C} 4-\mathrm{C} 3-\mathrm{N} 3-\mathrm{O} 32$ & $8.9(2)$ & $\mathrm{C} 6-\mathrm{C} 1-\mathrm{C} 11-\mathrm{O} 11$ & $-164.72(13)$ \\
\hline $\mathrm{C} 2-\mathrm{C} 3-\mathrm{N} 3-\mathrm{O} 32$ & $-169.88(14)$ & $\mathrm{O} 1-\mathrm{C} 11-\mathrm{O} 11-\mathrm{C} 12$ & $-1.7(2)$ \\
\hline $\mathrm{C} 4-\mathrm{C} 3-\mathrm{N} 3-\mathrm{O} 31$ & $-172.16(15)$ & $\mathrm{C} 1-\mathrm{C} 11-\mathrm{O} 11-\mathrm{C} 12$ & $177.03(12)$ \\
\hline $\mathrm{C} 2-\mathrm{C} 3-\mathrm{N} 3-\mathrm{O} 31$ & $9.1(2)$ & $\mathrm{C} 11-\mathrm{O} 11-\mathrm{C} 12-\mathrm{C} 21$ & $93.65(16)$ \\
\hline $\mathrm{C} 2-\mathrm{C} 3-\mathrm{C} 4-\mathrm{C} 5$ & $-0.6(2)$ & $\mathrm{O} 11-\mathrm{C} 12-\mathrm{C} 21-\mathrm{C} 22$ & 89.93 (17) \\
\hline $\mathrm{N} 3-\mathrm{C} 3-\mathrm{C} 4-\mathrm{C} 5$ & $-179.32(13)$ & $\mathrm{O} 11-\mathrm{C} 12-\mathrm{C} 21-\mathrm{C} 26$ & $-89.78(17)$ \\
\hline $\mathrm{C} 3-\mathrm{C} 4-\mathrm{C} 5-\mathrm{C} 6$ & $0.2(2)$ & $\mathrm{C} 26-\mathrm{C} 21-\mathrm{C} 22-\mathrm{C} 23$ & $-0.7(2)$ \\
\hline $\mathrm{C} 3-\mathrm{C} 4-\mathrm{C} 5-\mathrm{N} 5$ & $-178.99(13)$ & $\mathrm{C} 12-\mathrm{C} 21-\mathrm{C} 22-\mathrm{C} 23$ & $179.61(15)$ \\
\hline $\mathrm{C} 4-\mathrm{C} 5-\mathrm{N} 5-\mathrm{O} 51$ & $7.5(2)$ & $\mathrm{C} 21-\mathrm{C} 22-\mathrm{C} 23-\mathrm{C} 24$ & $0.2(3)$ \\
\hline $\mathrm{C} 6-\mathrm{C} 5-\mathrm{N} 5-\mathrm{O} 51$ & $-171.70(14)$ & $\mathrm{C} 22-\mathrm{C} 23-\mathrm{C} 24-\mathrm{C} 25$ & $0.6(3)$ \\
\hline $\mathrm{C} 4-\mathrm{C} 5-\mathrm{N} 5-\mathrm{O} 52$ & $-173.68(14)$ & $\mathrm{C} 23-\mathrm{C} 24-\mathrm{C} 25-\mathrm{C} 26$ & $-0.8(3)$ \\
\hline $\mathrm{C} 6-\mathrm{C} 5-\mathrm{N} 5-\mathrm{O} 52$ & $7.1(2)$ & $\mathrm{C} 24-\mathrm{C} 25-\mathrm{C} 26-\mathrm{C} 21$ & $0.3(3)$ \\
\hline $\mathrm{C} 4-\mathrm{C} 5-\mathrm{C} 6-\mathrm{C} 1$ & $0.2(2)$ & $\mathrm{C} 22-\mathrm{C} 21-\mathrm{C} 26-\mathrm{C} 25$ & $0.4(2)$ \\
\hline $\mathrm{N} 5-\mathrm{C} 5-\mathrm{C} 6-\mathrm{C} 1$ & $179.36(13)$ & $\mathrm{C} 12-\mathrm{C} 21-\mathrm{C} 26-\mathrm{C} 25$ & $-179.86(15)$ \\
\hline $\mathrm{C} 2-\mathrm{C} 1-\mathrm{C} 6-\mathrm{C} 5$ & $-0.2(2)$ & & \\
\hline
\end{tabular}

Hydrogen-bond geometry $\left(A,{ }^{\circ}\right)$

\begin{tabular}{lllll}
\hline$D-\mathrm{H} \cdots A$ & $D-\mathrm{H}$ & $\mathrm{H} \cdots A$ & $D \cdots A$ & $D-\mathrm{H} \cdots A$ \\
\hline $\mathrm{C} 2-\mathrm{H} 2 \cdots \mathrm{O} 32^{\mathrm{i}}$ & 0.95 & 2.52 & $3.460(2)$ & 170 \\
$\mathrm{C} 6-\mathrm{H} 6 \cdots \mathrm{O} 1^{\mathrm{ii}}$ & 0.95 & 2.57 & $3.493(2)$ & 164 \\
$\mathrm{C} 12-\mathrm{H} 12 A \cdots \mathrm{O} 52^{\mathrm{ii}}$ & 0.99 & 2.56 & $3.474(2)$ & 154 \\
$\mathrm{C} 22-\mathrm{H} 22 \cdots \mathrm{O} 51^{\mathrm{iii}}$ & 0.95 & 2.57 & $3.360(2)$ & 140 \\
\hline
\end{tabular}

Symmetry codes: (i) $-y+1, x-1 / 2, z+1 / 2$; (ii) $-x+1,-y+1,-z$; (iii) $-y+1, x-1 / 2, z+3 / 2$. 